\title{
Heat and Mass Transfer in the Boundary Layer of Unsteady Viscous Nanofluid along a Vertical Stretching Sheet
}

\author{
Eshetu Haile and B. Shankar \\ Department of Mathematics, Osmania University, Hyderabad 500 007, India \\ Correspondence should be addressed to Eshetu Haile; eshetuhg@gmail.com
}

Received 18 July 2014; Accepted 22 November 2014; Published 18 December 2014

Academic Editor: Fu-Yun Zhao

Copyright ( 2014 E. Haile and B. Shankar. This is an open access article distributed under the Creative Commons Attribution License, which permits unrestricted use, distribution, and reproduction in any medium, provided the original work is properly cited.

\begin{abstract}
Heat and mass transfer in the boundary-layer flow of unsteady viscous nanofluid along a vertical stretching sheet in the presence of magnetic field, thermal radiation, heat generation, and chemical reaction are presented in this paper. The sheet is situated in the $x z$ plane and $y$ is normal to the surface directing towards the positive $y$-axis. The sheet is continuously stretching in the positive $x$-axis and the external magnetic field is applied to the system parallel to the positive $y$-axis. With the help of similarity transformations, the partial differential equations are transformed into a couple of nonlinear ordinary differential equations. The new problem is then solved numerically by a finite-difference scheme known as the Keller-box method. Effects of the necessary parameters in the flow field are explicitly studied and briefly explained graphically and in tabular form. For the selected values of the pertinent parameters appearing in the governing equations, numerical results of velocity, temperature, concentration, skin friction coefficient, Nusselt number, and Sherwood number are obtained. The results are compared to the works of others (from previously published journals) and they are found in excellent agreement.
\end{abstract}

\section{Introduction}

The flow over a stretching surface is an important problem in many engineering processes with applications in industries such as extrusion, melt-spinning, hot rolling, wire drawing, glass-fiber production, manufacture of plastic and rubber sheets, and cooling of a large metallic plate in a bath, which may be an electrolyte. In industry, polymer sheets and filaments are manufactured by continuous extrusion of the polymer from a die to a windup roller, which is located at a finite distance away. The thin polymer sheet constitutes a continuously moving surface with a nonuniform velocity through an ambient fluid [1]. Bachok et al. [2] studied boundary layer flow of nanofluids over a moving surface in a flowing fluid and, moreover, a study on boundary layer flow of a nanofluid past a stretching sheet with a convective boundary condition was conducted by Makinde and Aziz [3]. Olanrewaju et al. [4] examined boundary layer flow of nanofluids over a moving surface in a flowing fluid in the presence of radiation. An analysis of mixed convection heat transfer from a vertical continuously stretching sheet has been presented by Chen [5].

In many practical situations the material moving in a quiescent fluid is due to the fluid flow induced by the motion of the solid material and/or by the thermal buoyancy. Therefore, the resulting flow and the thermal field are determined by these two mechanisms, that is, surface motion and thermal buoyancy. It is well known that the buoyancy force stemming from the heating or cooling of the continuous stretching sheet alters the flow and the thermal fields and thereby the heat transfer characteristics of the manufacturing processes [6]. Effects of thermal buoyancy on the flow and heat transfer over a stretching sheet were reported by many researchers. Chen and Strobel [7] investigated buoyancy effects in boundary layer adjacent to a continuous moving horizontal flat plate. Karwe and Jaluria [8] showed that the thermal buoyancy effects are more prominent when the plate moves vertically, that is, aligned with the gravity, than when it is horizontal. Ali [9] examined the buoyancy effect on the boundary layer induced by continuous surface stretched with rapidly decreasing velocities. Buoyancy driven heat and mass transfer 
over a stretching sheet in a porous medium with radiation and ohmic heating was studied by Dulal and Hiranmoy [10]. AboEldahab and El Aziz [11] presented the problem of steady, laminar, hydromagnetic heat transfer by mixed convection over an inclined stretching surface in the presence of spaceand temperature dependent heat generation or absorption effects. Ali and Al-Yousef $[12,13]$ investigated the problem of laminar mixed convection adjacent to a moving vertical surface with suction or injection. On the other hand, Khan et al. [14] studied the unsteady free convection boundary layer flow of a nanofluid along a stretching sheet with thermal radiation and viscous dissipation effects in the presence of a magnetic field.

The study of MHD boundary layer flow on a continuous stretching sheet has attracted considerable attention during the last few decades due to its numerous applications in industrial manufacturing processes. In particular, the metallurgical processes such as drawing, annealing, and tinning of copper wires involve cooling of continuous strips or filaments by drawing them through a quiescent fluid. Controlling the rate of cooling in these processes can affect the properties of the final product. Thus, rate of cooling can be greatly controlled by the use of electrically conducting fluid and the application of the magnetic field [15]. Magnetic field effects on free convection flow of a nanofluid past a vertical semi-infinite flat plate was studied by Hamad et al. [16]. Effects of a thin gray fluid on MHD free convective flow near a vertical plate with ramped wall temperature under small magnetic Reynolds number [17] and free convective oscillatory flow and mass transfer past a porous plate in the presence of radiation of an optically thin fluid [18] have been studied. Moreover, MHD Flow and heat transfer over stretching/shrinking sheets with external magnetic field, viscous dissipation, and joule effects were studied by Jafar et al. [19].

Radiative heat transfer in which heat is transmitted from one point to another without heating the intervening medium has been found very important in the design of reliable equipment, nuclear plants, gas turbines, and various propulsion devices for aircraft, missiles, satellites, and space vehicles. Also, the effects of thermal radiation on the forced and free convection flows are important in the content of space technology and processes involving high temperature [10]. Influence of thermal radiation, viscous dissipation, and hall current on MHD convection flow over a stretched vertical flat plate was studied by Gnaneswara Reddy [20]. Vajravelu and Hadjinicolaou [21] examined heat transfer in a viscous fluid over a stretching sheet with viscous dissipation and internal heat generation. Hady et al. [22] analyzed the flow and heat transfer characteristics of a viscous nanofluid over a nonlinearly stretching sheet in the presence of thermal radiation. Postelnicu [23] studied the influence of chemical reaction on heat and mass transfer by natural convection from vertical surfaces in porous media considering Soret and Dufour effects. Shakhaoath Khan et al. [24] examined possessions of chemical reaction on MHD heat and mass transfer nanofluid flow on a continuously moving surface. Besides this, effects of chemical reactions, heat and mass transfer on nonlinear magnetohydrodynamic boundary layer

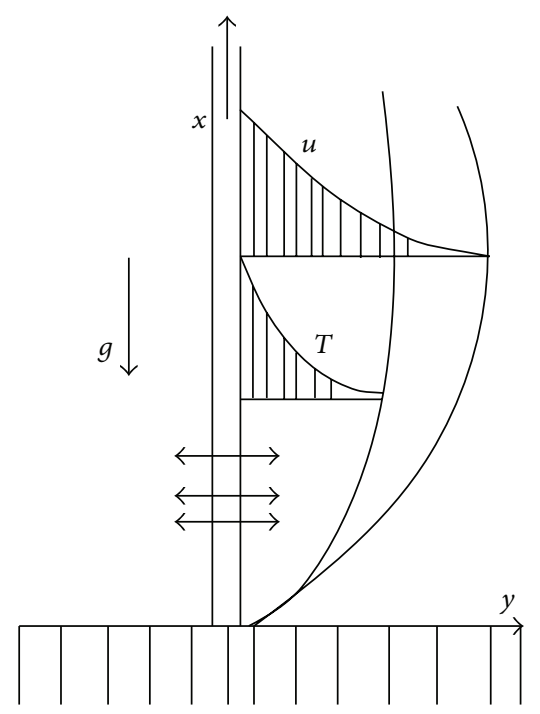

FIGURE 1: Physical model and coordinate system.

flow over a wedge with a porous medium in the presence of ohmic heating, and viscous dissipation were studied [25].

These days, because of the numerous applications of nanofluids in science and technology, a comprehensive study on heat and mass transfer in the boundary layer of unsteady viscous nanofluid in the presence of different fluid properties is indispensable. The paper entitled "Heat and mass transfer in the boundary layer of unsteady viscous nanofluid along a vertical stretching sheet" in the presence of thermal radiation, viscous dissipation, and chemical reaction is considered. Yet, the entitled paper has not been reported. Accordingly, we extended the works of Vendabai and Sarojamma [28] by incorporating viscous dissipation and chemical reaction terms in the energy and concentration equations, respectively, for more physical implications. The governing equations are reduced to a couple of nonlinear ODEs using similarity transformations; the resulting equations are solved numerically by using the Keller box. Effects of the pertinent parameters involved in the governing equations on velocity, temperature, concentration, skin friction, Nusselt number, and Sherwood number are briefly explained.

\section{Formulation of the Problem}

Unsteady two-dimensional boundary layer flow and heat transfer of a nanofluid along a stretching sheet coinciding with the plane $y=0$ are considered. The Cartesian coordinate system has its origin located at the leading edge of the sheet with the positive $x$-axis extending along the sheet in the upward direction, while the $y$-axis is measured normal to the surface of the sheet and is positive in the direction from the sheet to the fluid. A schematic representation of the physical model and coordinates system is shown in Figure 1. For $t<0$, it is assumed that the fluid and heat flows are steady but the unsteady scenario starts at $t=0$. We assume that the sheet is being stretched with the velocity $U_{w}(x, t)$ along the $x$-axis, keeping the origin fixed. Let $T_{w}(x, t)$ and $C_{w}(x, t)$ 
be the temperature and concentration of the sheet whereas let $T_{\infty}(x, t)$ and $C_{\infty}(x, t)$ be the ambient temperature and concentration, respectively. An external variable magnetic field $B=B_{0} / \sqrt{1-c t}$ is applied along the positive $y$ direction. The induced magnetic field is sufficiently weak to ignore magnetic induction effects; that is, magnetic Reynolds number is small. The charge density, external electrical field effects, and polarization voltage are ignored. Using Oberbeck Boussinesq approximation for buoyancy-driven flows, following Buongiorno [6, 15, 28-30], the governing equations for the flow become

$$
\begin{aligned}
& \frac{\partial u}{\partial x}+\frac{\partial v}{\partial y}=0 \\
& \frac{\partial u}{\partial t}+u \frac{\partial u}{\partial x}+v \frac{\partial u}{\partial y}=v \frac{\partial^{2} u}{\partial y^{2}}+\frac{g^{*}}{\rho_{f}} \\
& \times\left[\left(1-C_{\infty}\right) \rho_{f \infty} \beta\left(T-T_{\infty}\right)\right. \\
& \left.-\left(\rho_{p}-\rho_{f \infty}\right)\left(C-C_{\infty}\right)\right]-\frac{\sigma B^{2}}{\rho_{n f}} u,
\end{aligned}
$$

$$
\begin{aligned}
\frac{\partial T}{\partial t}+u \frac{\partial T}{\partial x}+v \frac{\partial T}{\partial y}= & \alpha \frac{\partial^{2} T}{\partial y^{2}}+\frac{\mu}{\rho C_{p}}\left(\frac{\partial u}{\partial y}\right)^{2} \\
& +\frac{Q}{\rho C_{p}}\left(T-T_{\infty}\right)-\frac{1}{\rho C_{p}} \frac{\partial q_{r}}{\partial y} \\
& +\tau\left[D_{B} \frac{\partial C}{\partial y} \frac{\partial T}{\partial y}+\frac{D_{T}}{T_{\infty}}\left(\frac{\partial T}{\partial y}\right)^{2}\right], \\
\frac{\partial C}{\partial t}+u \frac{\partial C}{\partial x}+v \frac{\partial C}{\partial y}=D_{B} \frac{\partial^{2} C}{\partial y^{2}}+\frac{D_{T}}{T_{\infty}} \frac{\partial^{2} T}{\partial y^{2}} & -K_{r}\left(C-C_{\infty}\right) .
\end{aligned}
$$

The boundary conditions associated to the differential equations are

$$
\begin{array}{r}
u=U_{w}(x, t), \quad v=V_{w}(x, t), \\
T=T_{w}(x, t), \quad C=C_{w}(x, t) \\
\text { at } y=0, \\
u \longrightarrow 0, \quad T \longrightarrow T_{\infty}, \quad C \longrightarrow C_{\infty} \\
\text { as } y \longrightarrow \infty,
\end{array}
$$

where $V_{w}(x, t)=-V_{0} / \sqrt{1-c t}$ is suction/injection velocity $\left(V_{0}>0\right.$ corresponds to suction velocity), $\tau=\left(\rho C_{p}\right)_{p} /\left(\rho C_{p}\right)_{f}$, $\alpha=k /\left(\rho C_{p}\right)_{f}, q_{r}$ is the radiative heat flux, and the heat generation coefficient $Q$ is defined by $Q=Q_{0} /(1-c t)$, where $Q_{0}$ represents the heat source if $Q_{0}>0$ and the heat sink if $Q_{0}<0$.

The continuous sheet moves in its own plane with the nonuniform velocity $U_{w}(x, t)=a x /(1-c t)$ where $a$ and $c$ are positive constants with dimensions(time $)^{-1}, a$ is the initial stretching rate, and $a /(1-c t)$ is the effective stretching rate which is increasing with time. $T_{w}(x, t)=T_{\infty}+$ $\left(b x /(1-c t)^{2}\right)$ is the temperature distribution of the sheet and the concentration distribution on the wall is given by $C_{w}(x, t)=C_{\infty}+\left(a x /(1-c t)^{2}\right)$ where $b$ is a constant and has a dimension (temperature/length) with $b>0$ and $b<0$ corresponds to the assisting and opposing flows and $b=0$ is forced convection limit (absence of buoyancy force). The expressions for $U_{w}(x, t), B(t), Q(t)$, and $V_{w}(x, t)$ are valid only for time $t<1 / c$ unless $c=0$.

Using the Rosseland diffusion approximation, the radiative heat flux $q_{r}$ is given by

$$
q_{r}=-\frac{4 \sigma^{*}}{3 k^{*}} \frac{\partial T^{4}}{\partial y} .
$$

We assume that the temperature differences within the flow are sufficiently small such that $T^{4}$ may be expressed as a linear function of temperature. Giving Taylor series expansion about $T_{\infty}$ and neglecting higher order terms we get

$$
T^{4} \approx 4 T_{\infty}^{3} T-3 T_{\infty}^{4} .
$$

Using (6) and (7) we obtain the expression $\partial q_{r} / \partial y$ as follows:

$$
\frac{\partial q_{r}}{\partial y}=-\frac{16 \sigma^{*} T_{\infty}^{3}}{3 k^{*}} \frac{\partial^{2} T}{\partial y^{2}} .
$$

In order to transform the governing equations into a system of ordinary differential equations, we introduce the following nondimensional quantities into (1)-(4):

$$
\begin{gathered}
\eta=\left(\frac{a}{v(1-c t)}\right)^{1 / 2} y, \\
T=T_{\infty}+\left(T_{w}-T_{\infty}\right) g(\eta), \\
C=C_{\infty}+\left(C_{w}-C_{\infty}\right) h(\eta), \\
\psi=\left(\frac{v a}{1-c t}\right)^{1 / 2} x f(\eta),
\end{gathered}
$$

where the stream function $\psi$ is defined by $u=\partial \psi / \partial y$ and $v=$ $-\partial \psi / \partial x$ which identically satisfies the continuity equation (1). Substitution of the similarity variables into (2)-(4) gives

$$
\begin{aligned}
& f^{\prime \prime \prime}+f f^{\prime \prime}-f^{\prime 2}-A\left(f^{\prime}+\frac{1}{2} \eta f^{\prime \prime}\right) \\
& \quad+\Lambda(g-N r h)-M^{2} f^{\prime}=0, \\
& (1+R) g^{\prime \prime}+N b g^{\prime} h^{\prime}+N t g^{\prime 2} \\
& \quad-\operatorname{Pr}\left(f^{\prime} g+A\left(2 g+\frac{1}{2} \eta g^{\prime}\right)-f g^{\prime}-\gamma g-E c f^{\prime \prime^{2}}\right)=0,
\end{aligned}
$$

$$
\begin{aligned}
h^{\prime \prime} & +\frac{N t}{N b} g^{\prime \prime} \\
& -\operatorname{Le}\left(A\left(2 h+\frac{1}{2} \eta h^{\prime}\right)+f^{\prime} h-f h^{\prime}+\operatorname{Re}_{x} \xi h\right)=0,
\end{aligned}
$$


and the corresponding boundary conditions become

$$
\begin{aligned}
f(0)=f w, \quad f^{\prime}(0)=1, & \\
g(0)=1, \quad h(0)=1, & \\
f^{\prime}(\eta) \longrightarrow 0, \quad g(\eta) \longrightarrow 0, & h(\eta) \longrightarrow 0, \\
& \text { as } \eta \longrightarrow \infty .
\end{aligned}
$$

Primes denote differentiation with respect to $\eta, f w=V_{0} / \sqrt{a v}$ (suction/injection parameter), and the various parameters are given by $\gamma=Q_{0} / a \rho C_{p}$ (heat source parameter), $\mathrm{Nb}=$ $\left(\tau D_{B}\left(C_{w}-C_{\infty}\right)\right) / \alpha$ (Brownian motion parameter), $\mathrm{Nt}=$ $\left(\tau D_{T}\left(T_{w}-T_{\infty}\right)\right) / \alpha T_{\infty}$ (thermophoresis parameter), $\operatorname{Re}_{x}=$ $x U_{w} / v$ (local Reynolds number), $A=c / a$ (unsteady parameter), $\Lambda=\left(b g^{*} \beta\left(1-C_{\infty}\right)\right) / a^{2}$ (free convection parameter), $M^{2}=\sigma B_{0}^{2} / a \rho_{f}$ (magnetic parameter), $\xi=v K_{r} / U_{w}^{2}$ (scaled chemical reaction parameter), $R=16 \sigma^{*} T_{\infty}^{3} / 3 k^{*} k_{\infty}$ (radiation parameter), $\mathrm{Nr}=\left(\rho_{p}-\rho_{f \infty}\right)\left(C_{w}-C_{\infty}\right) /(\beta(1-$ $\left.C_{\infty}\right) \rho_{f \infty}\left(T_{w}-T_{\infty}\right)$ ) (buoyancy ratio number), $\operatorname{Pr}=v / \alpha$ (Prandtl number), Ec $=U_{w}^{2} /\left(C_{p}\left(T_{w}-T_{\infty}\right)\right)$ (Eckert number), and Le $=v / D_{B}$ (Lewis number).

We are interested to study the skin-friction coefficient $C_{f}$, the local Nusselt number $\mathrm{Nu}_{x}$, and the local Sherwood number $\mathrm{Sh}_{x}$. These quantities are defined as

$$
\begin{gathered}
C_{f}=\frac{2 \tau_{w}}{\rho U_{w}^{2}}, \\
\mathrm{Nu}_{x}=\frac{x q_{w}}{k_{\infty}\left(T_{w}-T_{\infty}\right)}, \\
\mathrm{Sh}_{x}=\frac{x J_{w}}{D_{B}\left(C_{w}-C_{\infty}\right)},
\end{gathered}
$$

where $\tau_{w}, q_{w}$, and $J_{w}$ are the skin friction, heat flux, and mass flux at the surface and these quantities are, respectively, defined by

$$
\begin{gathered}
\tau_{w}=-\mu\left(\frac{\partial u}{\partial y}\right)_{y=0}, \\
q_{w}=-k_{\infty}(1+R)\left(\frac{\partial T}{\partial y}\right)_{y=0}, \\
J_{w}=-D_{B}\left(\frac{\partial C}{\partial y}\right)_{y=0} .
\end{gathered}
$$

Using (14) and (15), the dimensionless skin friction coefficient (surface drag), wall heat, and mass transfer rates become

$$
\begin{gathered}
\sqrt{\mathrm{Re}_{x}} C_{f}=-2 f^{\prime \prime}(0), \\
\frac{\mathrm{Nu}_{x}}{\sqrt{\mathrm{Re}_{x}}}=-(1+R) g^{\prime}(0), \\
\frac{\mathrm{Sh}_{x}}{\sqrt{\mathrm{Re}_{x}}}=-h^{\prime}(0),
\end{gathered}
$$

where $\operatorname{Re}_{x}=x U_{w} / v$ is the local Reynolds number.
It is important to mention that if $A=0$, the problem under consideration is reduced to the steady state flow scenario; the absence of the viscous and chemical reaction terms in this paper reduces it to the works of Vendabai and Sarojamma [28]. It is also worth mentioning that the absence of $M, \mathrm{Nr}, \mathrm{Nt}, \mathrm{Nb}, \gamma, \mathrm{Ec}$, and $R$ from (10) and (11) together with the impermeability condition of the sheet corresponds to the works of Ishak et al. [27] whereas the absence of these parameters except $\mathrm{Nr}$ and letting $\epsilon=0$ in their paper corresponds to the works of Vajravelu et al. [6]. Assigning $A=0, \Lambda=0$ and the absence of $M, R, \mathrm{Nt}, \mathrm{Nb}, \gamma$, and $\mathrm{Ec}$ in (10) and (11) reduces these equations to those of Grubka and Bobba [26] with $\gamma=1$ in their paper; in this case the problem has the closed form solution as given in the cited journal.

\section{Numerical Solution}

As (10)-(12) are nonlinear, it is impossible to get the closed form solutions. Consequently, the equations with the boundary conditions (13) are solved numerically by means of a finite-difference scheme known as the Keller-box method, as mentioned by Cebeci and Bradshaw [31]. According to Vajravelu et al. [6], the principal steps in the Keller box method to get the numerical solutions are the following:

(i) reduce the given ODEs to a system of first order equations;

(ii) write the reduced ODEs to finite differences;

(iii) linearize the algebraic equations by using Newton's method and write them in vector form;

(iv) solve the linear system by the block tridiagonal elimination technique.

One of the factors affecting the accuracy of the method is the appropriateness of the initial guesses. The following initial guesses are chosen:

$$
\begin{gathered}
f_{0}(\eta)=1+f_{w}-e^{-\eta}, \\
g_{0}(\eta)=e^{-\eta}, \quad h_{0}(\eta)=e^{-\eta} .
\end{gathered}
$$

In this study a uniform grid of size $\Delta \eta=0.006$ is taken and the solutions are obtained with an error of tolerance $10^{-5}$ in all cases, which gives about four decimal places accurate for most of the prescribed quantities as shown in all tables.

\section{Results and Discussion}

Our main objective in this flow scenario is to investigate the effects of unsteadiness on the flow quantities of the nanofluid. In order to investigate the flow quantities like velocity, temperature, concentration, and so forth a parametric study has taken place to illustrate effects of the various parameters like magnetic parameter, unsteady parameter, suction parameter, viscous dissipation parameter, buoyancy ratio number, heat source parameter, radiation parameter, Prandtl number, free convection parameter, chemical reaction parameter, Lewis number, thermophoresis parameter, and Brownian motion parameter upon the nature of flow 


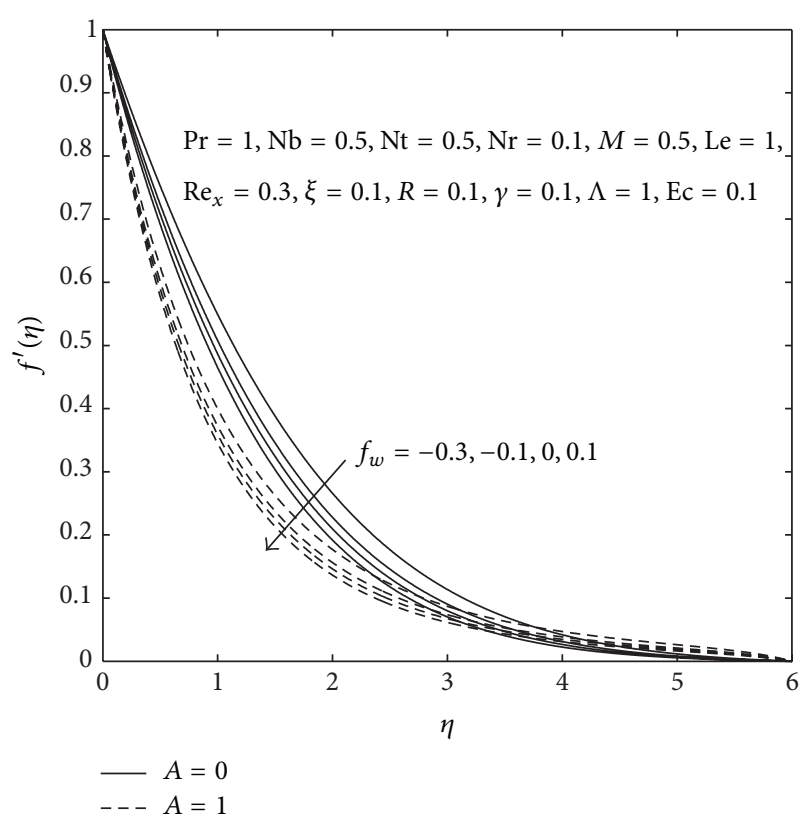

Figure 2: Effects of $f_{w}$ and $A$ on velocity profile.

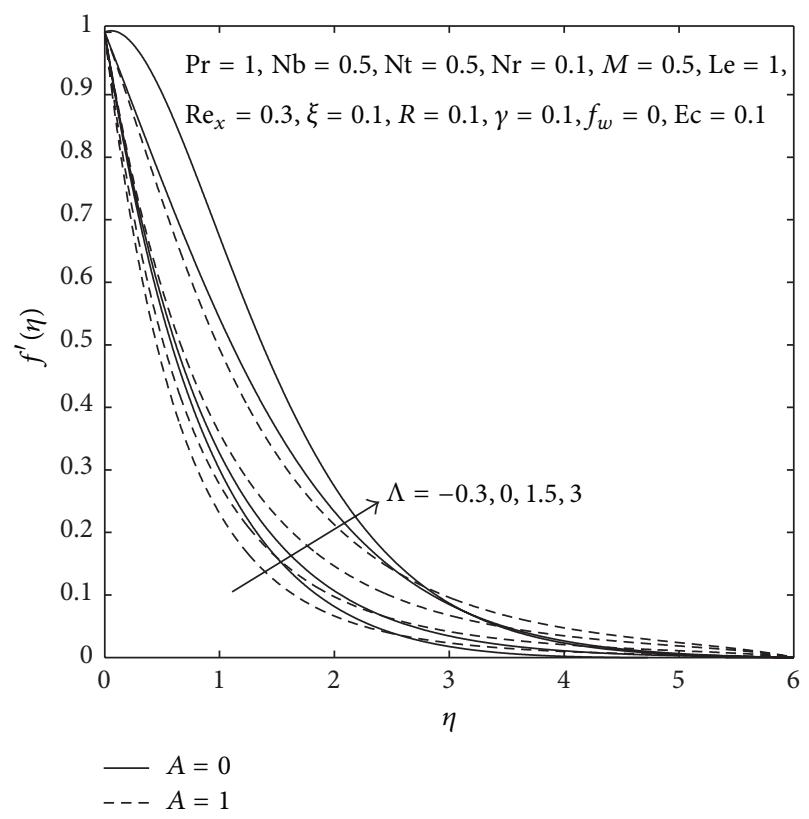

Figure 3: Effects of $\Lambda$ and $A$ on velocity profile.

and transport; the numerical results are displayed graphically (from Figure 2 to Figure 31) and in tables (from Table 1 to Table 5). The present results for temperature gradient are compared to the works of Grubka and Bobba [26], Ishak et al. [27], Vendabai and Sarojamma [28], and Vajravelu et al. [6] and they are found in good agreement as shown in Table 1.

The velocity, temperature, concentration, skin friction coefficient, wall heat, and mass transfer rates for some prescribed values of the various parameters $\mathrm{Pr}, \mathrm{Nb}, \mathrm{Nt}, \Lambda$, $R, \mathrm{Ec}, \mathrm{Nr}, \mathrm{Le}, A, \gamma, \xi, M, f w$, and $\operatorname{Re}_{x}$ are briefly presented,

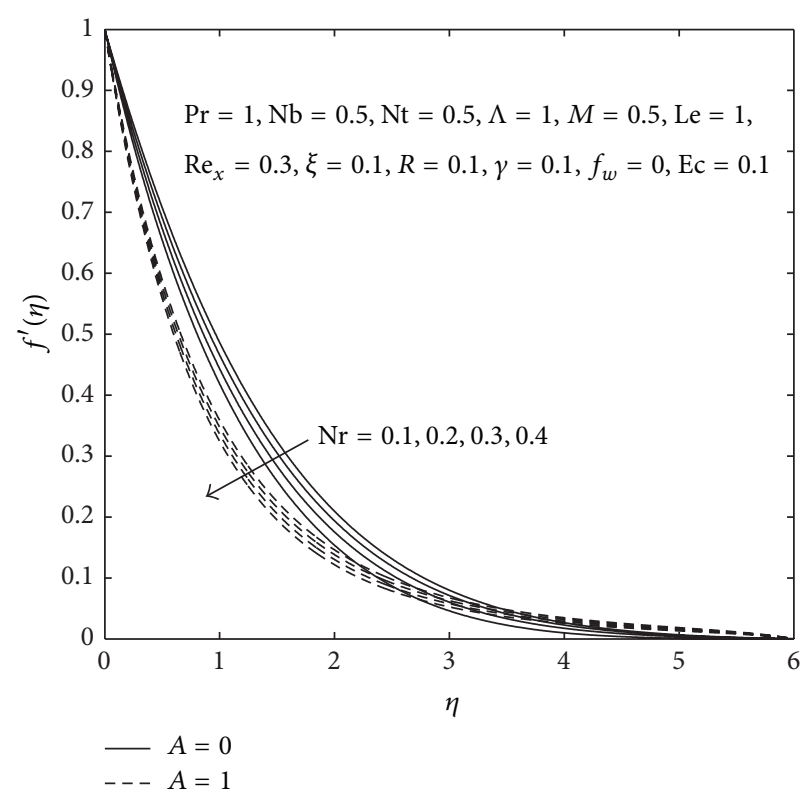

Figure 4: Effects of $\mathrm{Nr}$ and $A$ on velocity profile.

explained, and interpreted graphically and in table form. The velocity, temperature, and concentration profiles are clearly illustrated graphically whereas the skin friction coefficient, wall heat, and mass transfer rates for the pertinent parameters are tabulated. First, let us see effects of these parameters on velocity, temperature, and concentration graphically.

Figure 2 illustrates effects of suction and unsteady parameters on velocity profile. The suction parameter retards the velocity profile both in the steady and in the unsteady cases. It is certain that the role of unsteady parameter is to reduce the velocity profile. It is observed that when the surface is impermeable $\left(f_{w}=0\right)$, the velocity in the steady flow case reduces to zero faster (see Figures 3-10). As we move away from the boundary (as the distance $\eta$ from the boundary increases), the velocity of the nanofluid continuously declines to zero. It is found that as the free convection parameter $\Lambda$ increases, the velocity profile in both the steady and the unsteady cases increase significantly (Figure 3 ). It is worth mentioning that the free convection parameter $\Lambda>0$ corresponds to the heating of the fluid (assisting flow), $\Lambda<$ 0 corresponds to cooling of the fluid (opposing flow), and $\Lambda=0$ means the absence of free convection currents [28]. An increase in $\Lambda$ brings about the enhancement of velocity due to the enhancement of convection currents and thus the momentum boundary layer thickness increases.

Figure 4 depicts effects of buoyancy ratio number $\mathrm{Nr}$ and unsteady parameter $A$ on velocity profile. The buoyancy parameter opposes the velocity profile both in the steady and in the unsteady cases whereas the Brownian motion parameter $\mathrm{Nb}$ enhances the velocity profile significantly both in the steady and in the unsteady cases. Figure 5 shows the effect of Brownian motion parameter and unsteady parameter on velocity profile. 
TABLE 1: Comparison of some of the values of wall temperature gradient $-g^{\prime}(0)$ obtained by the cited researchers and the present results for $M=R=\mathrm{Nb}=\mathrm{Nt}=\mathrm{Nr}=\mathrm{Ec}=\gamma=f_{w}=0$.

\begin{tabular}{|c|c|c|c|c|c|c|c|}
\hline A & $\Lambda$ & $\operatorname{Pr}$ & $\begin{array}{l}\text { Grubka and } \\
\text { Bobba [26] }\end{array}$ & Ishak et al. [27] & $\begin{array}{l}\text { Vajravelu } \\
\text { et al. [6] }\end{array}$ & $\begin{array}{c}\text { Vendabai and } \\
\text { Sarojamma } \\
{[28]}\end{array}$ & $\begin{array}{l}\text { Present } \\
\text { study }\end{array}$ \\
\hline 0 & 0 & 1 & 1.0000 & 1.0000 & 1.000000 & 1.000484 & 1.000484 \\
\hline 0 & 0 & 3 & 1.9237 & 1.9237 & 1.923687 & 1.923455 & 1.923457 \\
\hline 0 & 0 & 10 & 3.7207 & 3.7207 & 3.720788 & 3.720514 & 3.720557 \\
\hline 0 & 0 & 100 & 12.2940 & 12.2941 & 12.30039 & 12.293708 & 12.296229 \\
\hline 0 & 1 & 1 & - & 1.0873 & 1.087206 & 1.087082 & 1.087082 \\
\hline 0 & 2 & 1 & - & 1.1423 & 1.142298 & 1.142201 & 1.142202 \\
\hline 0 & 3 & 1 & - & 1.1853 & 1.185197 & 1.185195 & 1.185197 \\
\hline 1 & 0 & 1 & - & 1.6820 & 1.681921 & 1.681992 & 1.681939 \\
\hline 1 & 1 & 1 & - & 1.7039 & 1.703910 & 1.703912 & 1.703358 \\
\hline
\end{tabular}

TABLE 2: Effects of free convection parameter $\Lambda$, unsteady parameter $A$, buoyancy ratio number $\mathrm{Nr}$, and magnetic parameter $M$ on skin friction coefficient, heat transfer, and mass transfer rates.

\begin{tabular}{|c|c|c|c|c|c|c|c|c|c|c|c|}
\hline & & & For $\mathrm{Nt}=$ & $\mathrm{Nb}=0$ & $\mathrm{r}=1, \mathrm{~L} \epsilon$ & $1, \mathrm{Ec}=0$. & $=0.1, R$ & $1, \xi=0$. & $e_{x}=0.1$, & & \\
\hline & amet & & & $A=0$ & & & $A=0.5$ & & & $A=1$ & \\
\hline$\Lambda$ & $\mathrm{Nr}$ & $M$ & $-f^{\prime \prime}(0)$ & $-g^{\prime}(0)$ & $-h^{\prime}(0)$ & $-f^{\prime \prime}(0)$ & $-g^{\prime}(0)$ & $-h^{\prime}(0)$ & $-f^{\prime \prime}(0)$ & $-g^{\prime}(0)$ & $-h^{\prime}(0)$ \\
\hline-0.1 & 0.1 & 0.5 & 1.1759 & 0.5781 & 0.6280 & 1.3099 & 0.9464 & 0.9536 & 1.4445 & 1.1837 & 1.1705 \\
\hline 1 & 0.1 & 0.5 & 0.6612 & 0.7042 & 0.7341 & 0.8990 & 0.9909 & 0.9884 & 1.0919 & 1.2118 & 1.1868 \\
\hline 1.5 & 0.1 & 0.5 & 0.4620 & 0.7331 & 0.7622 & 0.7246 & 1.0067 & 1.0018 & 0.9373 & 1.2228 & 1.1940 \\
\hline 3 & 0.1 & 0.5 & -0.0859 & 0.7926 & 0.8282 & 0.2300 & 1.0441 & 1.0381 & 0.4901 & 1.2503 & 1.2156 \\
\hline 1 & 0.2 & 0.5 & 0.7104 & 0.6951 & 0.7247 & 0.9416 & 0.9865 & 0.9845 & 1.1292 & 1.2089 & 1.1848 \\
\hline 1 & 0.3 & 0.5 & 0.7605 & 0.6852 & 0.7145 & 0.9845 & 0.9819 & 0.9805 & 1.1667 & 1.2059 & 1.1828 \\
\hline 1 & 0.4 & 0.5 & 0.8115 & 0.6744 & 0.7035 & 1.0279 & 0.9771 & 0.9764 & 1.2044 & 1.2028 & 1.1809 \\
\hline 1 & 0.1 & 0 & 0.5373 & 0.7246 & 0.7552 & 0.7874 & 1.0023 & 0.9990 & 0.9932 & 1.2196 & 1.1925 \\
\hline 1 & 0.1 & 0.5 & 0.6612 & 0.7042 & 0.7341 & 0.8990 & 0.9909 & 0.9884 & 1.0919 & 1.2118 & 1.1868 \\
\hline 1 & 0.1 & 1 & 0.9835 & 0.6477 & 0.6853 & 1.1914 & 0.9609 & 0.9646 & 1.3554 & 1.1910 & 1.1741 \\
\hline 1 & 0.1 & 1.5 & 1.4146 & 0.5670 & 0.6367 & 1.5885 & 0.9207 & 0.9408 & 1.7228 & 1.1623 & 1.1616 \\
\hline
\end{tabular}

TABLE 3: Effects of suction parameter $f_{w}$, unsteady parameter $A$, radiation parameter $R$, and Brownian motion parameter Nb on skin friction coefficient, heat transfer, and mass transfer rates.

\begin{tabular}{|c|c|c|c|c|c|c|c|c|c|c|c|}
\hline & & & or $\mathrm{Nt}=$ & $\mathrm{Nr}=0$. & $I=0.5$, & $=1, \mathrm{Le}=$ & $\mathrm{c}=0.1$, & $0.1, \lambda=$ & $=0.1, \mathrm{Re}_{\lambda}$ & & \\
\hline & amete & & & $A=0$ & & & $A=0.5$ & & & $A=1$ & \\
\hline$f_{w}$ & $\mathrm{Rd}$ & $\mathrm{Nb}$ & $-f^{\prime \prime}(0)$ & $-g^{\prime}(0)$ & $-h^{\prime}(0)$ & $-f^{\prime \prime}(0)$ & $-g^{\prime}(0)$ & $-h^{\prime}(0)$ & $-f^{\prime \prime}(0)$ & $-g^{\prime}(0)$ & $-h^{\prime}(0)$ \\
\hline-0.3 & 0.1 & 0.5 & 0.5365 & 0.6271 & 0.7005 & 0.7759 & 0.9135 & 0.9420 & 0.9688 & 1.1341 & 1.1361 \\
\hline-0.1 & 0.1 & 0.5 & 0.6164 & 0.6766 & 0.7337 & 0.8556 & 0.9637 & 0.9797 & 1.0489 & 1.1848 & 1.1752 \\
\hline 0 & 0.1 & 0.5 & 0.6607 & 0.7030 & 0.7002 & 0.8988 & 0.9901 & 0.9987 & 1.0917 & 1.2112 & 1.1949 \\
\hline 0.1 & 0.1 & 0.5 & 0.7079 & 0.7305 & 0.7667 & 0.9444 & 1.0172 & 1.0176 & 1.1365 & 1.2382 & 1.2147 \\
\hline 0 & 0 & 0.5 & 0.6712 & 0.7266 & 0.7284 & 0.9066 & 1.0224 & 0.9760 & 1.0982 & 1.2505 & 1.1689 \\
\hline 0 & 0.5 & 0.5 & 0.6266 & 0.6277 & 0.8188 & 0.8726 & 0.8859 & 1.0703 & 1.0701 & 1.0847 & 1.2767 \\
\hline 0 & 1 & 0.5 & 0.5957 & 0.5613 & 0.8775 & 0.8478 & 0.7927 & 1.1324 & 1.0494 & 0.9714 & 1.3472 \\
\hline 0 & 1.5 & 0.5 & 0.5728 & 0.5130 & 0.9190 & 0.8285 & 0.7240 & 1.1769 & 1.0332 & 0.8877 & 1.3974 \\
\hline 0 & 0.1 & 0.5 & 0.6607 & 0.7030 & 0.7002 & 0.8988 & 0.9901 & 0.9987 & 1.0917 & 1.2112 & 1.1949 \\
\hline 0 & 0.1 & 1 & 0.6342 & 0.5905 & 0.9760 & 0.8771 & 0.8468 & 1.2747 & 1.0733 & 1.0423 & 1.5192 \\
\hline 0 & 0.1 & 3 & 0.5617 & 0.3023 & 1.1170 & 0.8162 & 0.4717 & 1.4363 & 1.0209 & 0.5964 & 1.7054 \\
\hline 0 & 0.1 & 5 & 0.5140 & 0.1761 & 1.1413 & 0.7737 & 0.2926 & 1.4573 & 0.9838 & 0.3771 & 1.7265 \\
\hline
\end{tabular}


TABLE 4: Effects of thermophoresis parameter Nt, unsteady parameter $A$, Prandtl number Pr and heat source parameter $\gamma$ on skin friction coefficient, heat transfer and mass transfer rates.

\begin{tabular}{|c|c|c|c|c|c|c|c|c|c|c|c|}
\hline & & & For $\mathrm{Nb}$ & $5, \mathrm{Nr}=$ & $M=0.5, \mathrm{I}$ & $=1, E c=$ & $R=0.1$, & $1, \xi=0$ & $e_{x}=0.3$ & & \\
\hline & Iram & & & $A=0$ & & & $A=0.5$ & & & $A=1$ & \\
\hline $\mathrm{Nt}$ & $\mathrm{Pr}$ & $\gamma$ & $-f^{\prime \prime}(0)$ & $-g^{\prime}(0)$ & $-h^{\prime}(0)$ & $-f^{\prime \prime}(0)$ & $-g^{\prime}(0)$ & $-h^{\prime}(0)$ & $-f^{\prime \prime}(0)$ & $-g^{\prime}(0)$ & $-h^{\prime}(0)$ \\
\hline 0.5 & 1 & 0.1 & 0.6607 & 0.7030 & 0.7502 & 0.8988 & 0.9901 & 0.9987 & 1.0917 & 1.2112 & 1.1949 \\
\hline 1 & 1 & 0.1 & 0.6515 & 0.6423 & 0.4958 & 0.8916 & 0.9090 & 0.6836 & 1.0855 & 1.1139 & 0.8211 \\
\hline 3 & 1 & 0.1 & 0.6148 & 0.4826 & -0.0598 & 0.8629 & 0.6911 & 0.0462 & 1.0608 & 0.8503 & 0.0765 \\
\hline 5 & 1 & 0.1 & 0.5808 & 0.3958 & -0.2922 & 0.8377 & 0.5695 & -0.1931 & 1.0392 & 0.7016 & -0.1859 \\
\hline 0.5 & 0.7 & 0.1 & 0.6086 & 0.5643 & 0.8731 & 0.8579 & 0.8023 & 1.1335 & 1.0576 & 0.9849 & 1.3496 \\
\hline 0.5 & 1 & 0.1 & 0.6607 & 0.7030 & 0.7502 & 0.8988 & 0.9901 & 0.9987 & 1.0917 & 1.2112 & 1.1949 \\
\hline 0.5 & 1.5 & 0.1 & 0.7240 & 0.9032 & 0.5678 & 0.9466 & 1.2560 & 0.7954 & 1.1313 & 1.5303 & 0.9598 \\
\hline 0.5 & 2 & 0.1 & 0.7698 & 1.0783 & 0.4062 & 0.9804 & 1.4853 & 0.6116 & 1.1593 & 1.8045 & 0.7459 \\
\hline 0.5 & 1 & 0.2 & 0.6453 & 0.6548 & 0.7927 & 0.8918 & 0.9570 & 1.0228 & 1.0877 & 1.1847 & 1.2131 \\
\hline 0.5 & 1 & 0.3 & 0.6282 & 0.6037 & 0.8371 & 0.8842 & 0.9229 & 1.0473 & 1.0836 & 1.1577 & 1.2314 \\
\hline 0.5 & 1 & 0.4 & 0.6091 & 0.5491 & 0.8837 & 0.8762 & 0.8878 & 1.0722 & 1.0792 & 1.1301 & 1.2499 \\
\hline
\end{tabular}

TABLE 5: Effects of viscous dissipation parameter Ec, unsteady parameter $A$, Lewis number Le, and chemical reaction parameter $\xi$ on skin friction coefficient, heat transfer, and mass transfer rates.

\begin{tabular}{|c|c|c|c|c|c|c|c|c|c|c|c|}
\hline & & & For $\mathrm{Nt}=$ & $5, \mathrm{Nb}=0$ & $\mathrm{Nr}=0.1$, & $=0.5, \operatorname{Pr}$ &,$\gamma=0.1$, & $=0.1, \Lambda$ & $\operatorname{Re}_{x}=0.3$ & & \\
\hline & ram & & & $A=0$ & & & $A=0.5$ & & & $A=1$ & \\
\hline $\mathrm{Ec}$ & Le & $\xi$ & $-f^{\prime \prime}(0)$ & $-g^{\prime}(0)$ & $-h^{\prime}(0)$ & $-f^{\prime \prime}(0)$ & $-g^{\prime}(0)$ & $-h^{\prime}(0)$ & $-f^{\prime \prime}(0)$ & $-g^{\prime}(0)$ & $-h^{\prime}(0)$ \\
\hline 0 & 1 & 0.5 & 0.6623 & 0.7138 & 0.8255 & 0.9005 & 1.0057 & 1.0426 & 1.0934 & 1.3209 & 1.2244 \\
\hline 0.2 & 1 & 0.5 & 0.6542 & 0.6801 & 0.8537 & 0.8946 & 0.9656 & 1.0739 & 1.0886 & 1.1843 & 1.2602 \\
\hline 0.5 & 1 & 0.5 & 0.6423 & 0.6320 & 0.8937 & 0.8860 & 0.9074 & 1.1192 & 1.0815 & 1.1159 & 1.3126 \\
\hline 1 & 1 & 0.5 & 0.6236 & 0.5580 & 0.9546 & 0.8721 & 0.8148 & 1.1910 & 1.0700 & 1.0055 & 1.3970 \\
\hline 0.1 & 1 & 0.5 & 0.6582 & 0.6967 & 0.8398 & 0.8975 & 0.9855 & 1.0583 & 1.0910 & 1.2075 & 1.2424 \\
\hline 0.1 & 2 & 0.5 & 0.6454 & 0.6590 & 1.4947 & 0.8860 & 0.9364 & 1.8485 & 1.0809 & 1.1498 & 2.1569 \\
\hline 0.1 & 3 & 0.5 & 0.6402 & 0.6389 & 1.9686 & 0.8809 & 0.9090 & 2.4243 & 1.0763 & 1.1173 & 2.8231 \\
\hline 0.1 & 4 & 0.5 & 0.6374 & 0.6258 & 2.3564 & 0.8780 & 0.8906 & 2.8965 & 1.0736 & 1.0952 & 3.3694 \\
\hline 0.1 & 1 & 0 & 0.6614 & 0.7048 & 0.7260 & 0.8992 & 0.9913 & 0.9832 & 1.0919 & 1.2121 & 1.1828 \\
\hline 0.1 & 1 & 0.5 & 0.6582 & 0.6967 & 0.8398 & 0.8975 & 0.9855 & 1.0583 & 1.0910 & 1.2075 & 1.2424 \\
\hline 0.1 & 1 & 1 & 0.6557 & 0.6902 & 0.9387 & 0.9861 & 0.9803 & 1.1289 & 1.0901 & 1.2032 & 1.2998 \\
\hline 0.1 & 1 & 1.5 & 0.6537 & 0.6848 & 1.0272 & 0.8949 & 0.9756 & 1.1956 & 1.0893 & 1.1991 & 1.3551 \\
\hline
\end{tabular}

Figures 6 and 7 show the effects of thermophoresis parameter $\mathrm{Nt}$ and thermal radiation parameter $R$, respectively, on velocity profile. The increments of both parameters assist the velocity profile to grow both in the steady and in the unsteady cases.

Figures 8 and 13 show effects of magnetic field on velocity and temperature profiles, respectively. The presence of magnetic field reduces the velocity throughout the boundary layer which is in conformity with the fact that the Lorentz force (magnetic force) acts as a retarding force and, consequently, it reduces the momentum boundary layer thickness significantly both in the steady and in the unsteady cases whereas it increases thermal boundary layer thickness. This happens because as the strength of the applied magnetic field increases in an electrically conducting fluid, it produces the resistive Lorentz force $\left(\sigma B^{2} / \rho\right) u$. This force decelerates the motion of the fluid in the boundary layer. On the other hand, we can define thermal energy as the additional work done required to drag the nanofluid against the action of the magnetic field $B$. The work done heats up the conducting nanofluid and upgrades the temperature profile. Thus, the presence of magnetic field in the flow regime decreases the momentum boundary layer thickness and enhances the thermal boundary layer thickness [32].

The presence of Prandit number also retards the velocity profile significantly both in the steady and in the unsteady flows as shown in Figure 9.

Figure 10 depicts the effect of heat source parameter $\gamma$ on velocity profile. This parameter enhances velocity of the flow significantly in the steady state situation whereas the enhancement of the parameter in the unsteady state situation is very minimal (see more on explanations for Figure 18).

Figure 11 shows the effect of suction parameter $f_{w}$ on temperature profile. The presence of suction in the flow decreases 


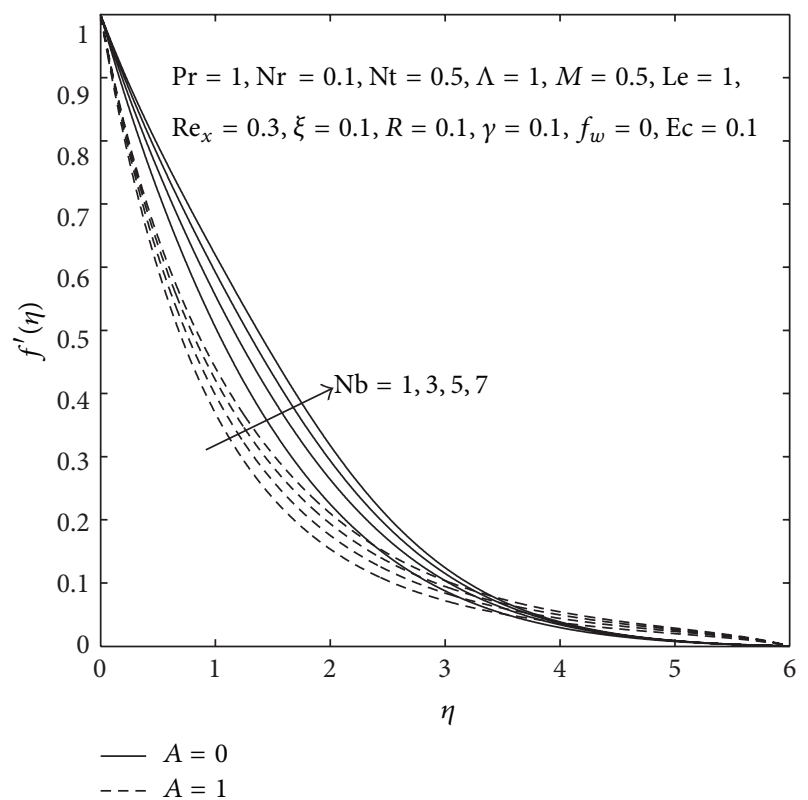

FIgURE 5: Effects of $\mathrm{Nb}$ and $A$ on velocity profile.

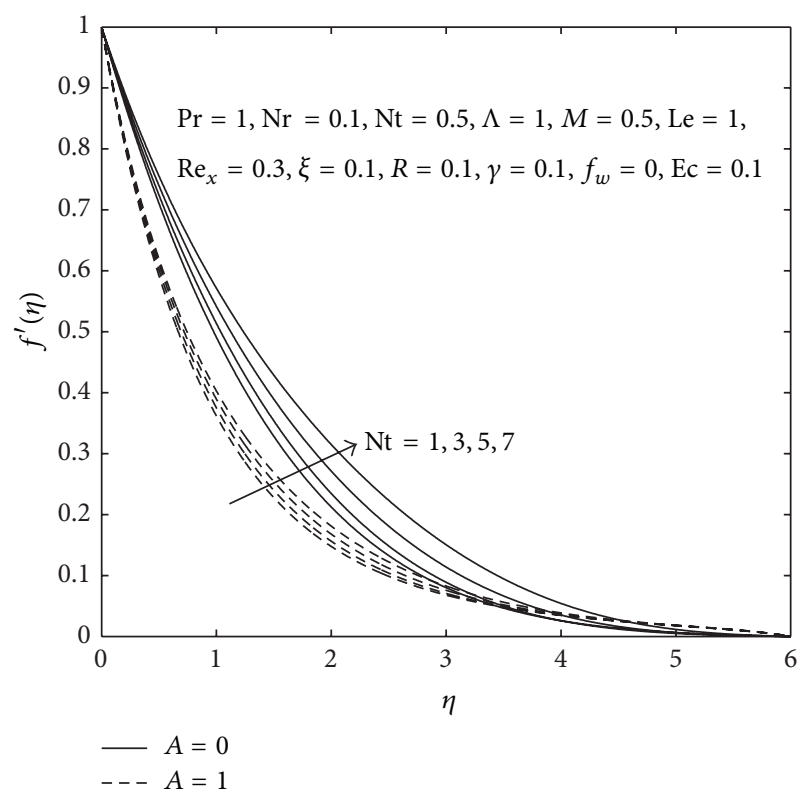

Figure 6: Effects of $\mathrm{Nt}$ and $A$ on velocity profile.

the temperature profile both in the steady and in the unsteady situations. It is obviously illustrated that the presence of unsteady parameter in a flow is to reduce the temperature profile. In addition to this, the free convection parameter $\Lambda$ significantly reduces the temperature profile both in the steady and in the unsteady state situations (see Figure 12). On the other hand, in the steady state flow scenario, the thermal boundary layer thickness grows considerably with increasing value of the Lorentz force. But the increment of the thermal boundary layer in the case of unsteady flow situations is nominal (see Figure 13).

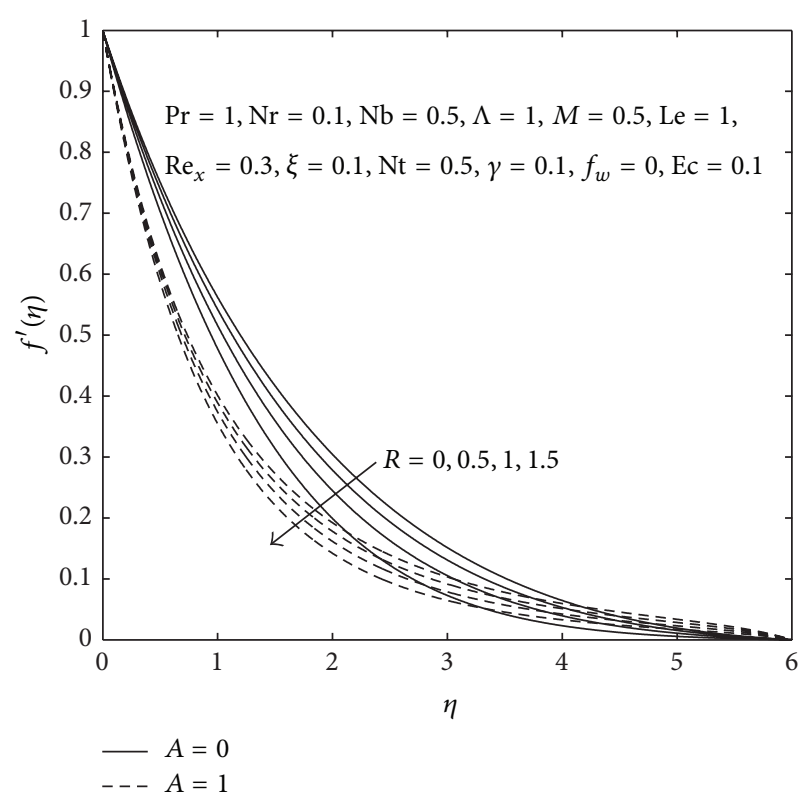

Figure 7: Effects of $R$ and $A$ on velocity profile.

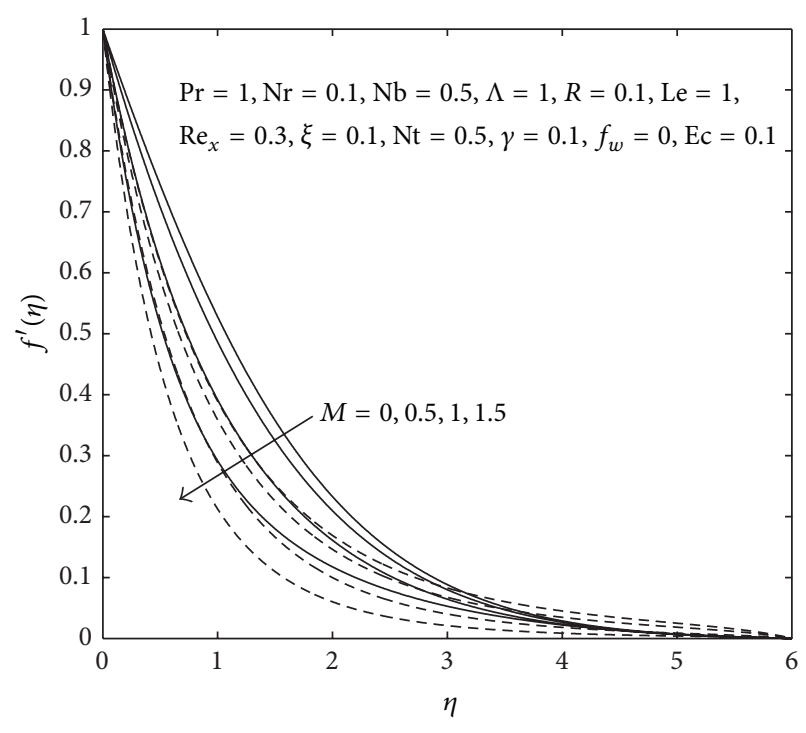

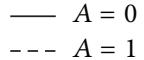

Figure 8: Effects of $M$ and $A$ on velocity profile.

Figures 14 and 15 show the effects of thermal radiation and Brownian motion parameters, respectively, on temperature. Both parameters significantly enhance the thickening of the thermal boundary layer thickness in the steady and unsteady state situations. As we move away from the boundary farther, the Brownian motion parameter makes the velocity decay to zero faster. This is due to the fact that thermal radiation inspires in thickening the thermal boundary layer at the expense of releasing heat energy from the flow region and it causes the system to cool. In reality this is true because 


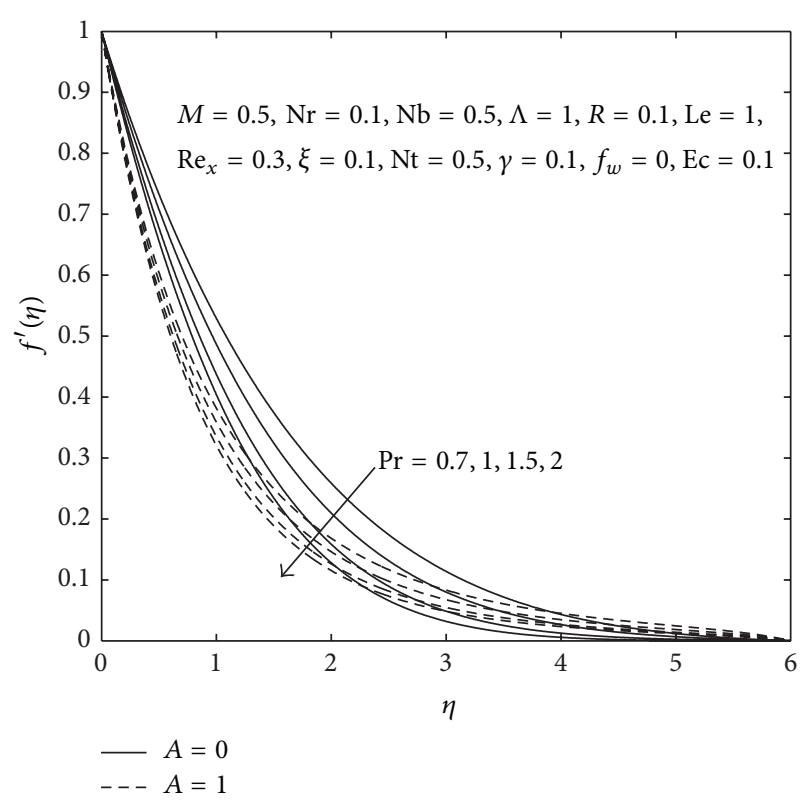

Figure 9: Effects of $\operatorname{Pr}$ and $A$ on velocity profile.

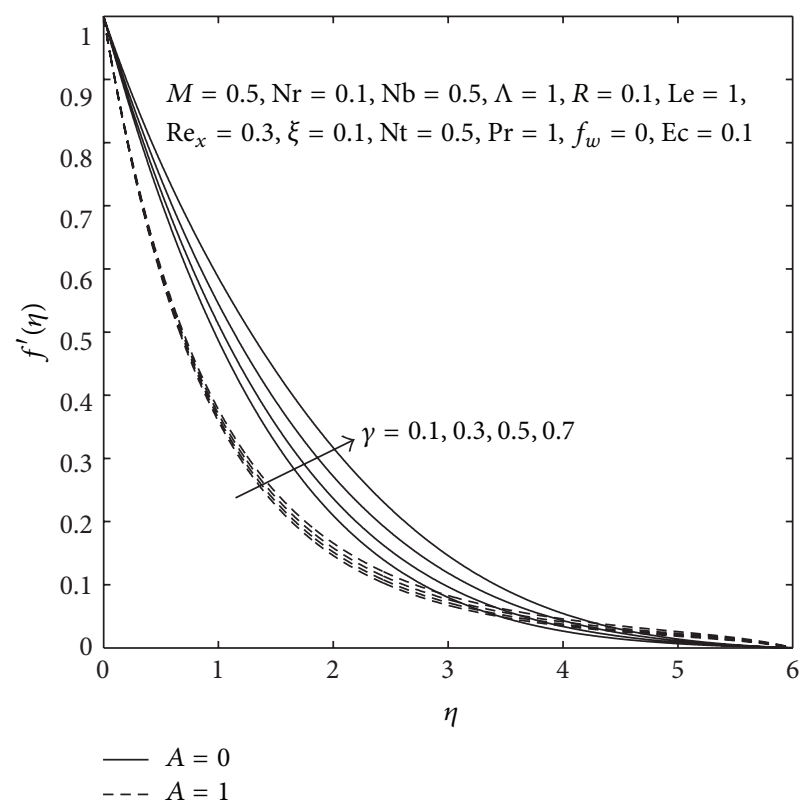

Figure 10: Effects of $\gamma$ and $A$ on velocity profile.

temperature increases as a result of increasing the Rosseland diffusion approximation for radiation $q_{r}$.

Figure 16 shows the effects of thermophoresis and unsteady parameters on temperature profile. In the steady state situation, thermophoresis parameter enhances the thickening of thermal boundary layer thickness considerably whereas the thickening of the boundary layer in the unsteady state situation is not significant. Figure 17 illustrates the effect of Eckert number Ec on temperature profile. As the viscous dissipation parameter increases, the temperature profiles of

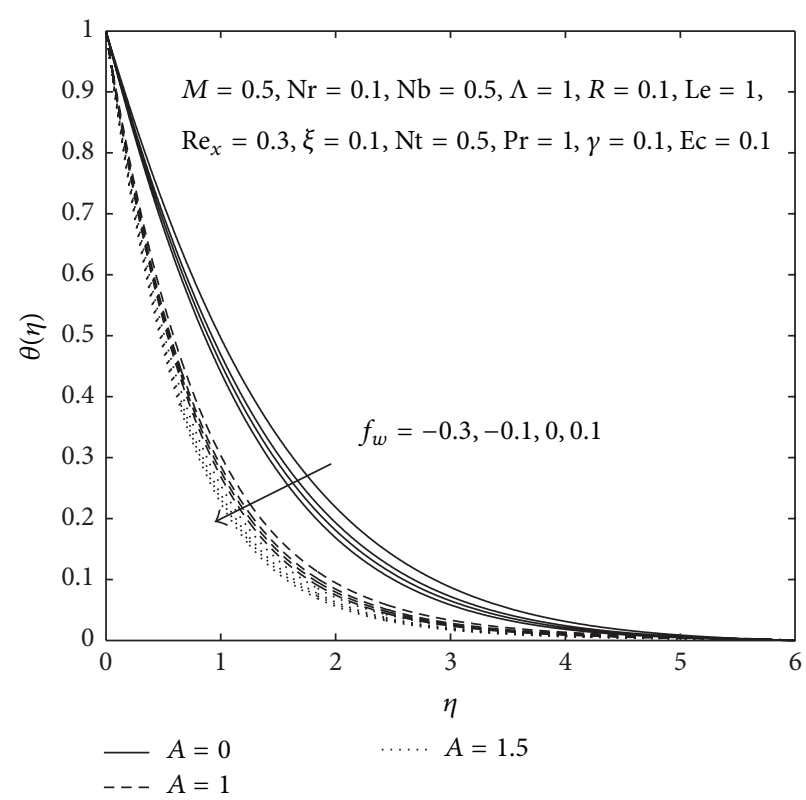

FIgURE 11: Effects of $f_{w}$ and $A$ on temperature profile.

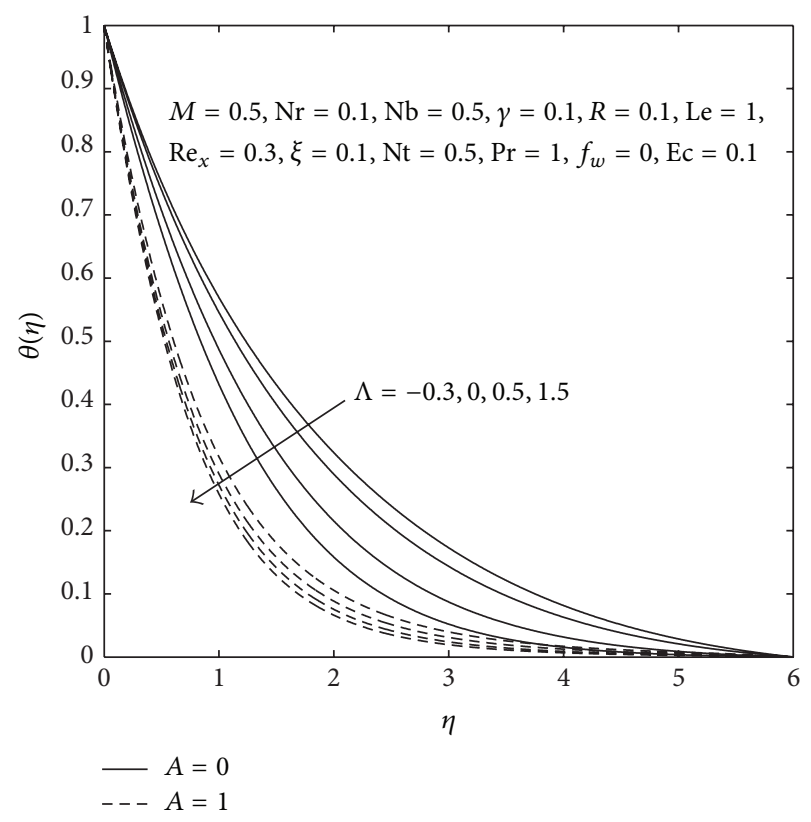

FIgURE 12: Effects of $\Lambda$ and $A$ on temperature profile.

both the steady and the unsteady state flows also increase but not significantly.

Figure 18 shows effects of the heat source parameter on temperature. Increasing the heat source parameter from 0.1 to 0.4 increases the temperature profile and hence it thickens the thermal boundary layer moderately in both the steady and the unsteady state flows. An increase in the values of heat source parameter $\gamma$ increases both the velocity and the temperature profiles. Because the presence of source of heat (in the flow regime) enhances thermal energy, as a result of this, temperature profile rises. The rise in temperature allows 


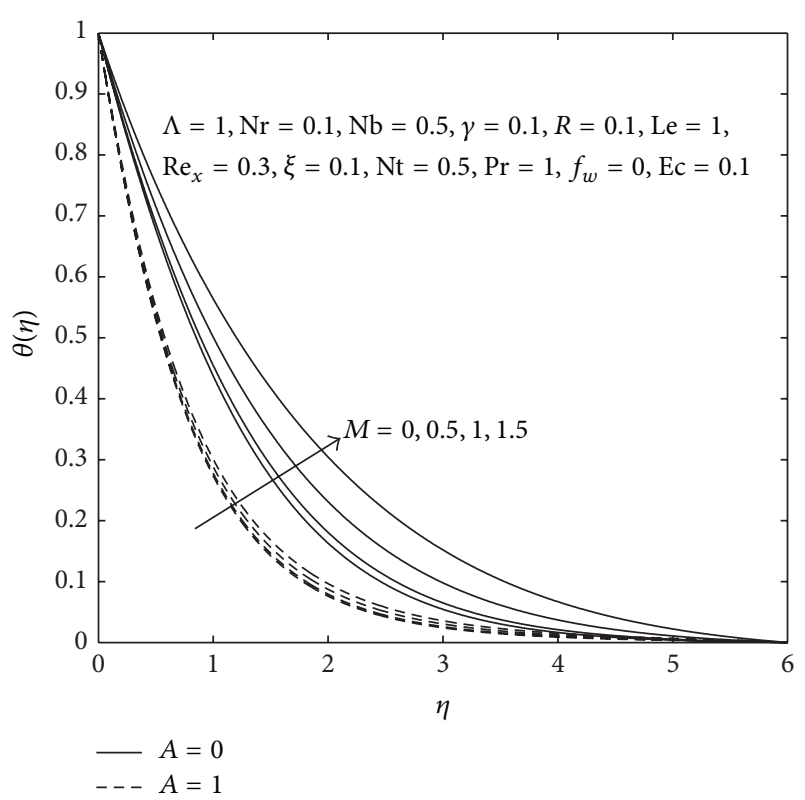

FIgURE 13: Effects of $M$ and $A$ on temperature profile.

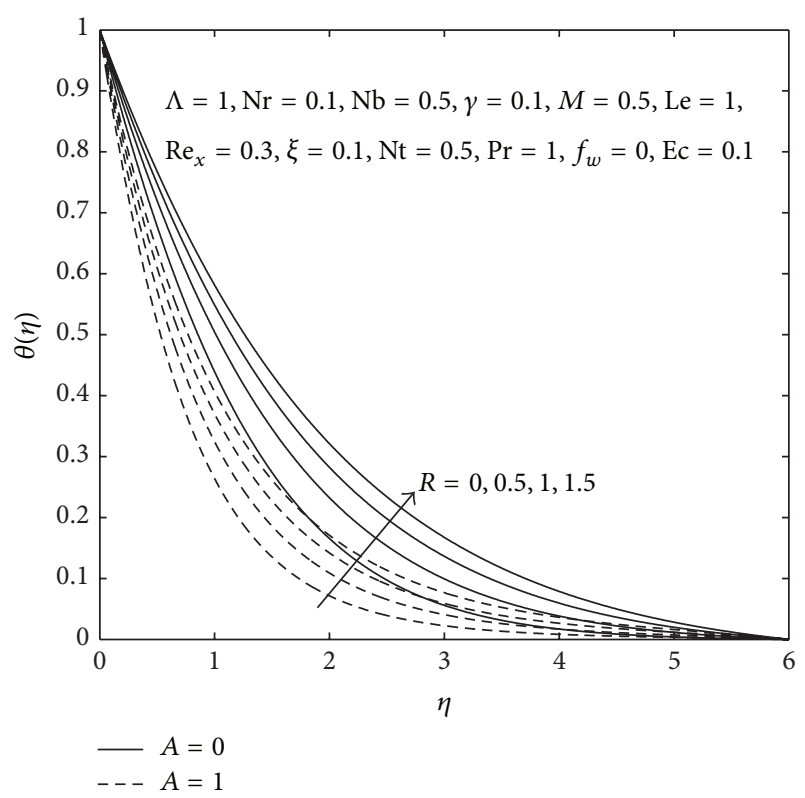

FIgURE 14: Effects of $R$ and $A$ on temperature profile.

the fluid to increase the velocity profile due to the effect of buoyancy; effects of heat sink on velocity and temperature profiles play oppositely.

As the buoyancy ratio number increases from 0 to 0.6 , the thermal boundary layer thickness increases in the case of steady state flows. For unsteady state situations, the effect of buoyancy ratio number on temperature is not significant as shown in Figure 19.

The effect of Prandtl number Pr on temperature is shown in Figure 20. This parameter significantly reduces the thermal boundary layer thickness in the cases of both steady and

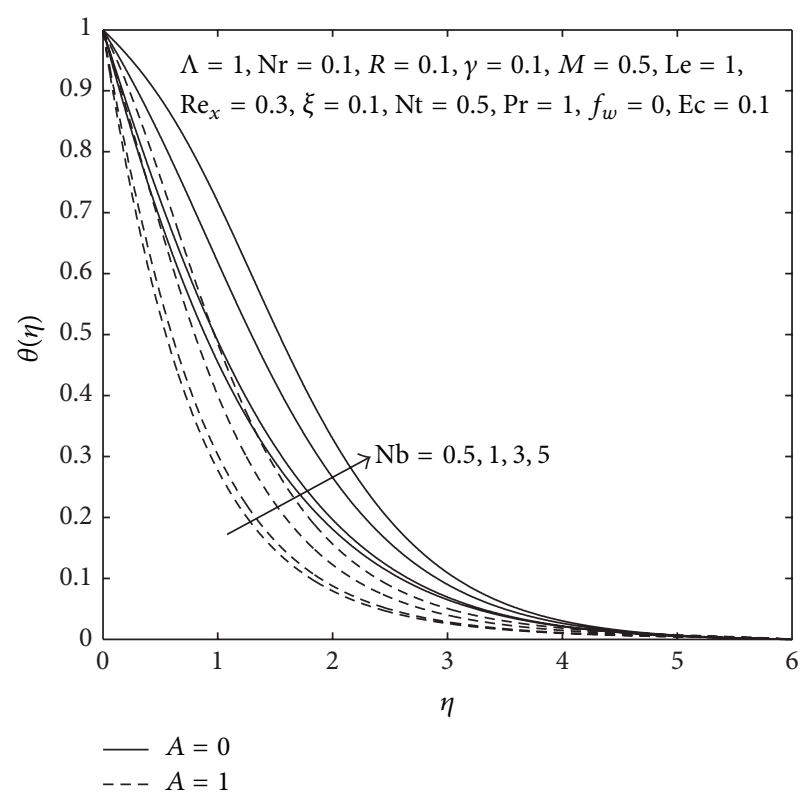

Figure 15: Effects of $\mathrm{Nb}$ and $A$ on temperature profile.

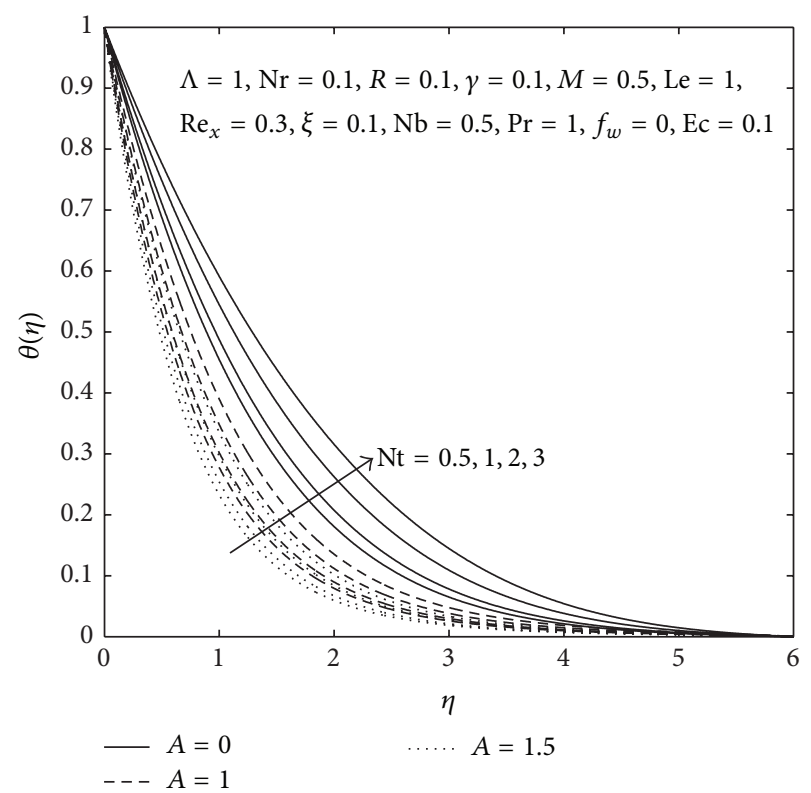

Figure 16: Effects of $\mathrm{Nt}$ and $A$ on temperature profile.

unsteady state situations. By definition, Prandtl number is a dimensionless number which is the ratio of momentum diffusivity to thermal diffusivity; that is, $\operatorname{Pr}=\mu C_{p} / k$. An increase in the values of $\operatorname{Pr}$ is equivalent to momentum diffusivity which dominates thermal diffusivity. Hence, thermal boundary layer thickness reduces as $\mathrm{Pr}$ increases. This is due to the fact that the larger the Prandtl number $\operatorname{Pr}$ is, the higher the viscosity (sticker) of the fluid and the thicker the momentum boundary layer will be compared to the thermal boundary layer. Consequently, heat transfer will be less convective. 


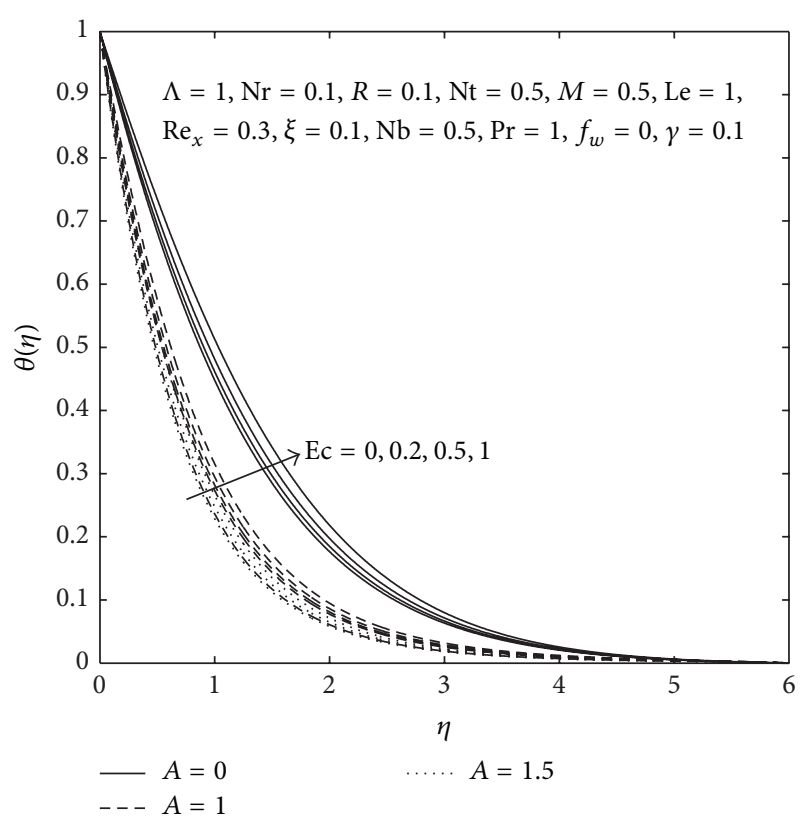

Figure 17: Effects of Ec and $A$ on temperature profile.

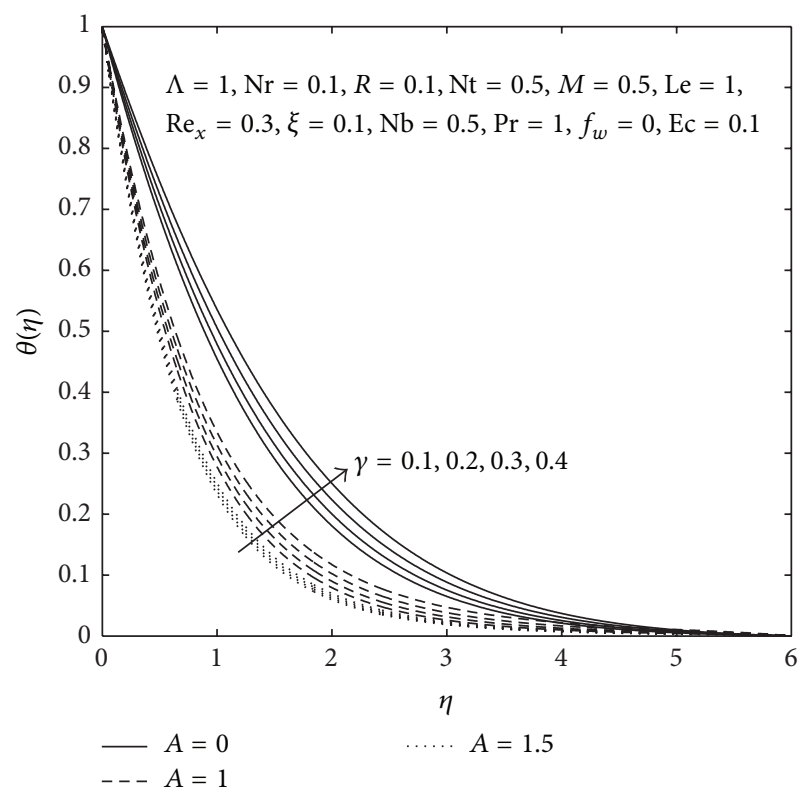

FIGURE 18: Effects of $\gamma$ and $A$ on temperature profile.

On the other hand, the presence of the free convection parameter $\Lambda$ minimizes nanoparticles volume fraction moderately in the cases of both steady and unsteady state flows (see Figure 21).

The effect of Brownian motion parameter on nanoparticles concentration is shown in Figure 22. As this parameter increases, nanoparticles volume fraction decreases both in the case of steady and in the case of unsteady state situations. Figure 23 shows the effect of thermophoresis parameter on nanoparticles volume fraction. It is observed from the figure that the concentration decays monotonically to zero as the

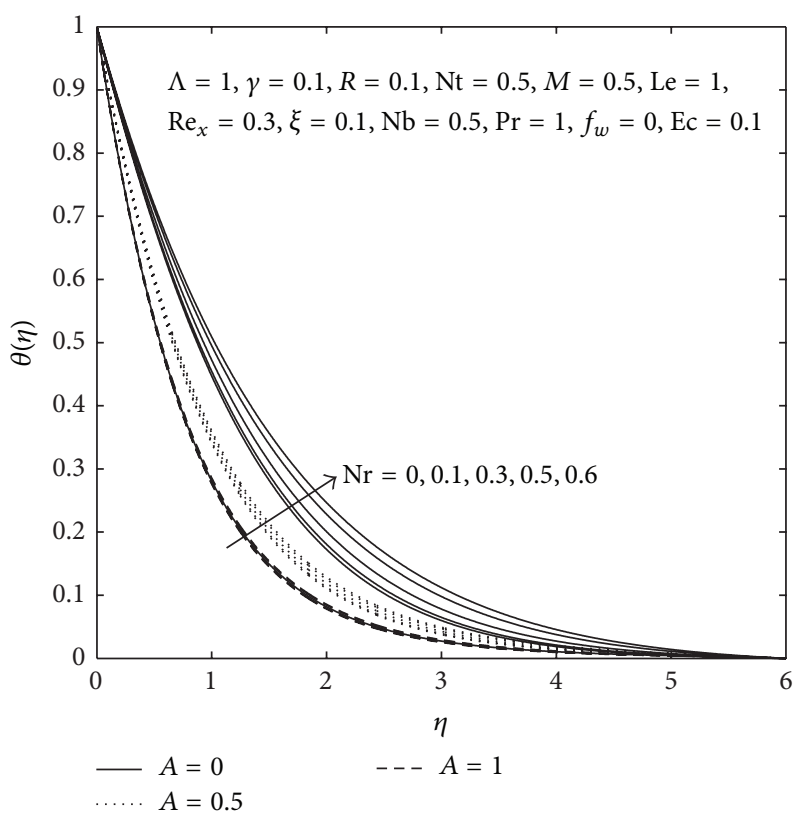

FIgURE 19: Effects of $\mathrm{Nr}$ and $A$ on temperature profile.

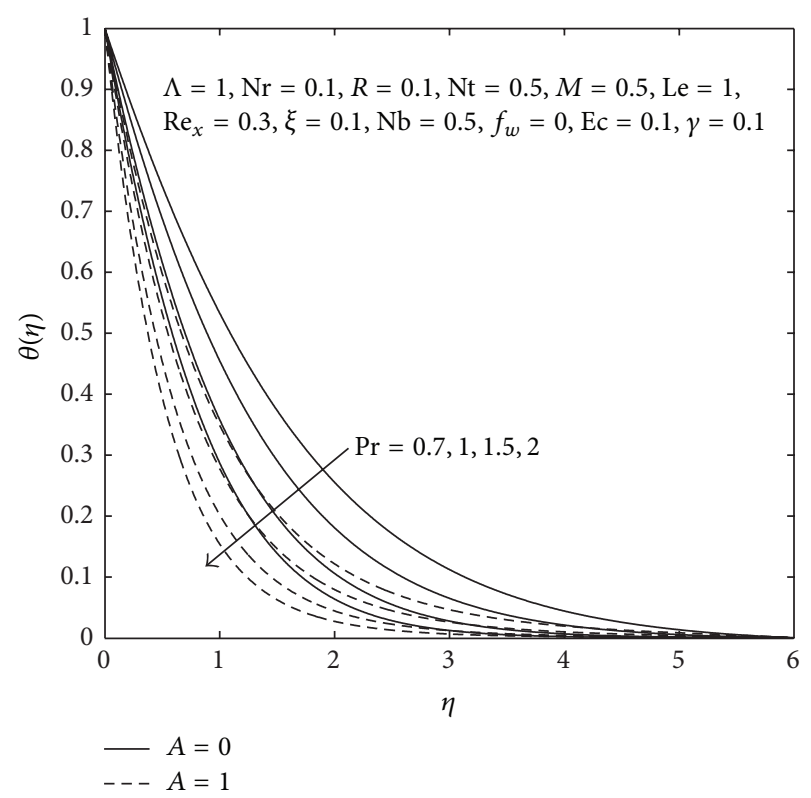

FIgURE 20: Effects of $\operatorname{Pr}$ and $A$ on temperature profile.

distance $\eta$ increases from the boundary. For larger values of $\mathrm{Nt}$ in the steady state situation, the concentration profile attains its maximum in the boundary layer and then declines to zero faster when we go farther. Increment of this parameter enhances the nanoparticles volume fraction strongly in both the steady and the unsteady state cases.

Effects of Prandtl number on concentration profile is depicted in Figure 24. Increasing the value of Prandtl number leads to an increase in the concentration profile of both the steady and the unsteady state cases. This increment ceases as we go farther from the boundary. The effect of Lewis number 


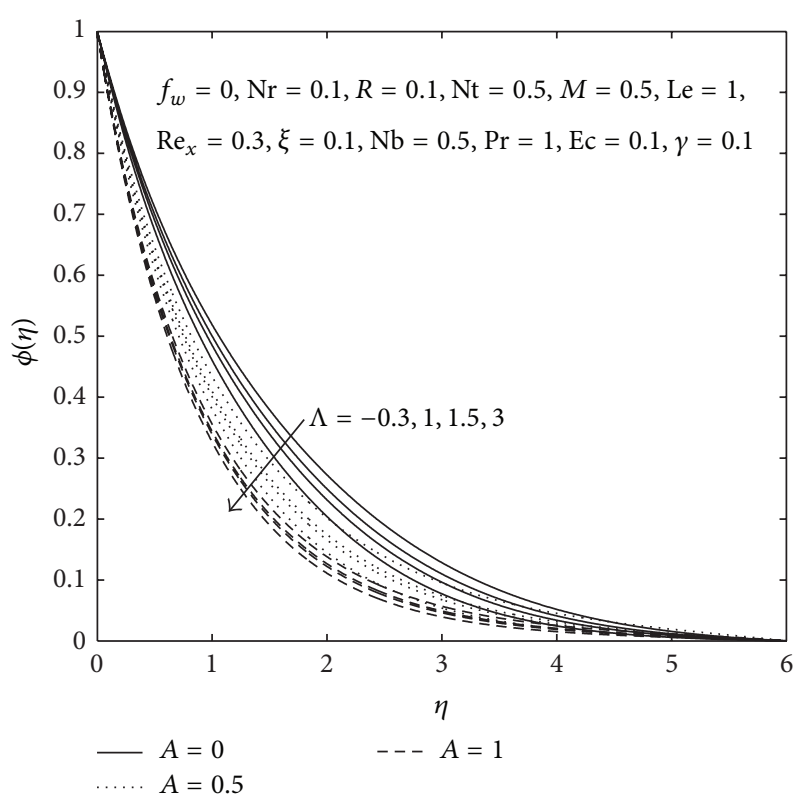

Figure 21: Effects of $\Lambda$ and $A$ on concentration profile.

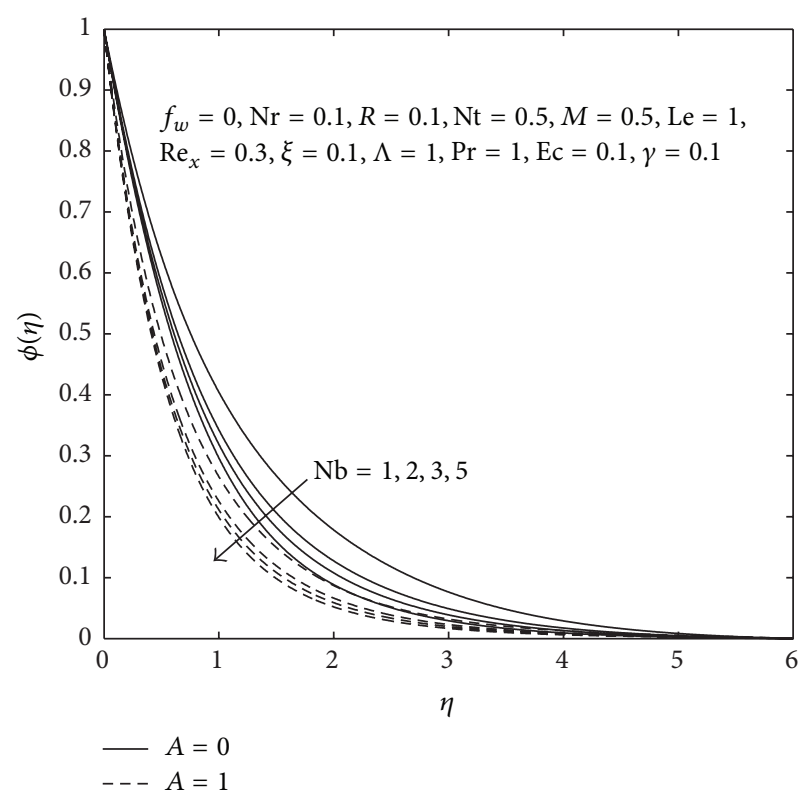

Figure 22: Effects of $\mathrm{Nb}$ and $A$ on concentration profile.

on concentration profile is shown in Figure 25. It is observed that increasing Lewis number significantly decreases the nanoparticles volume fraction in the case of both steady and unsteady state situations. Lewis number is a dimensionless number which is defined as the ratio of thermal diffusivity to mass diffusivity. Increasing the value of Le means increasing thermal boundary layer thickness at the expense of reducing the concentration boundary layer thickness; this leads to an increase in mass transfer rate.

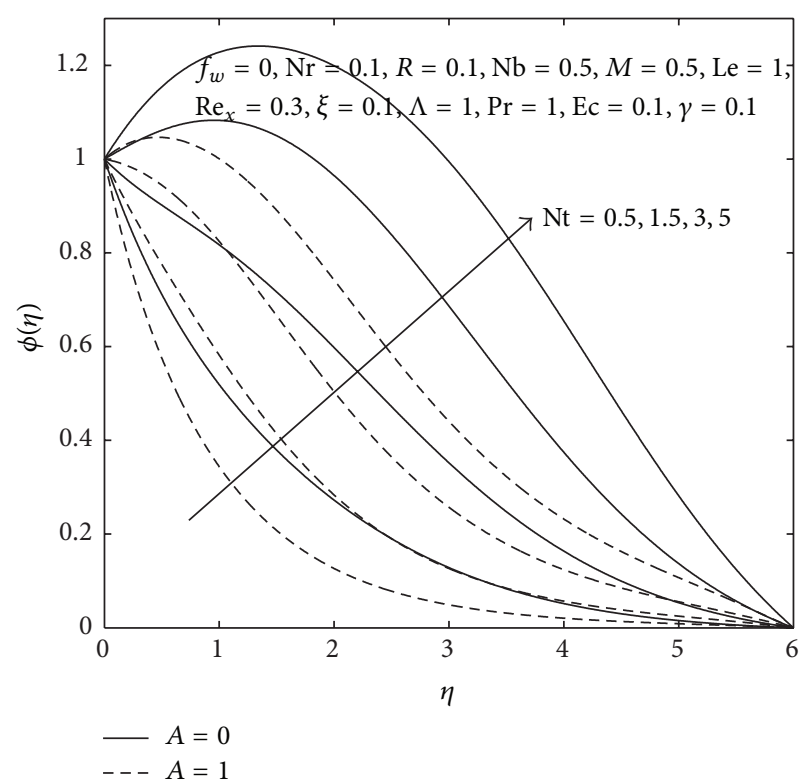

Figure 23: Effects of $\mathrm{Nt}$ and $A$ on concentration profile.

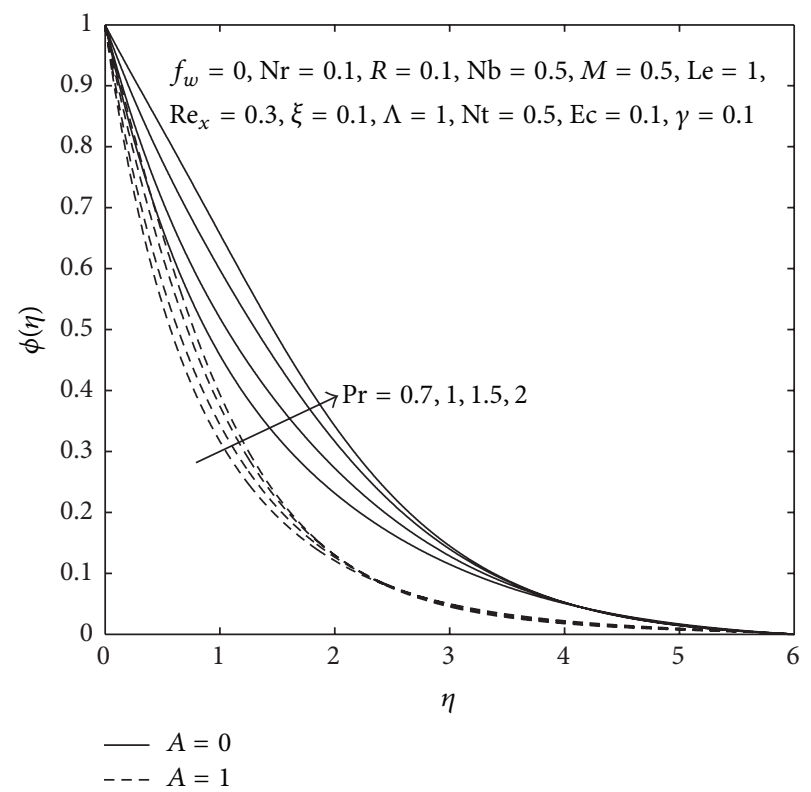

FIgURE 24: Effects of $\operatorname{Pr}$ and $A$ on concentration profile.

Figure 26 illustrates the influence of chemical reaction parameter on concentration profile. In the steady state situation, it is observed that the chemical reaction parameter enhances nanoparticles volume fraction but in the case of unsteady state situations, the effect of this parameter is minimal. It is understandable that the effect of unsteady parameter is to reduce the nanoparticle volume fraction. Moreover, the concentration decreases slowly as the suction parameter increases in the case of both the steady and unsteady state flow conditions (see Figure 27).

Figures 28 and 29 illustrate effects of magnetic parameter and buoyancy ratio number, respectively, on concentration 


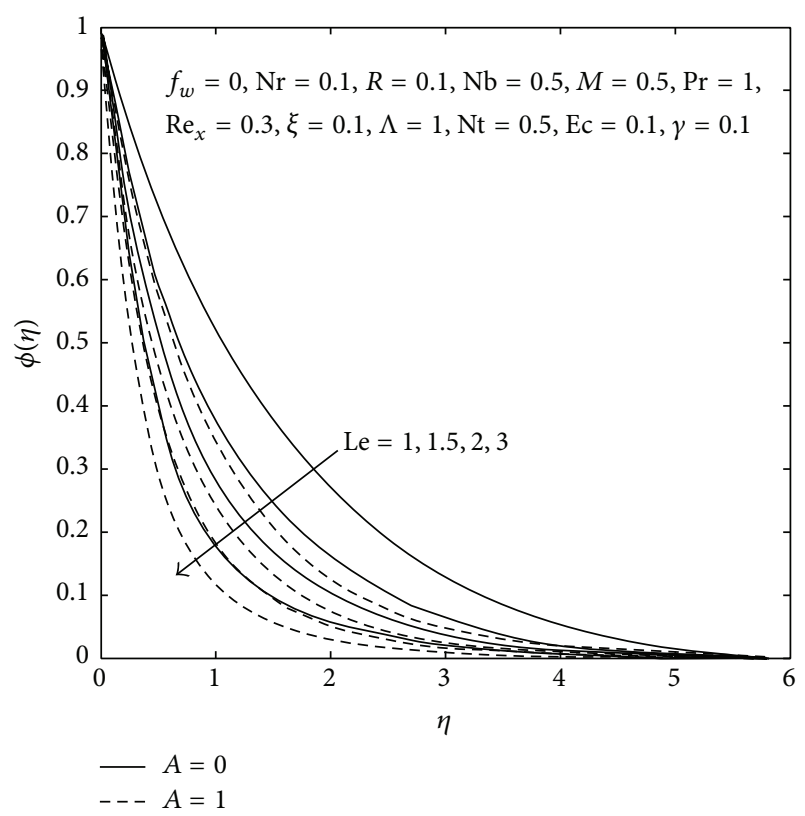

Figure 25: Effects of Le and $A$ on concentration profile.

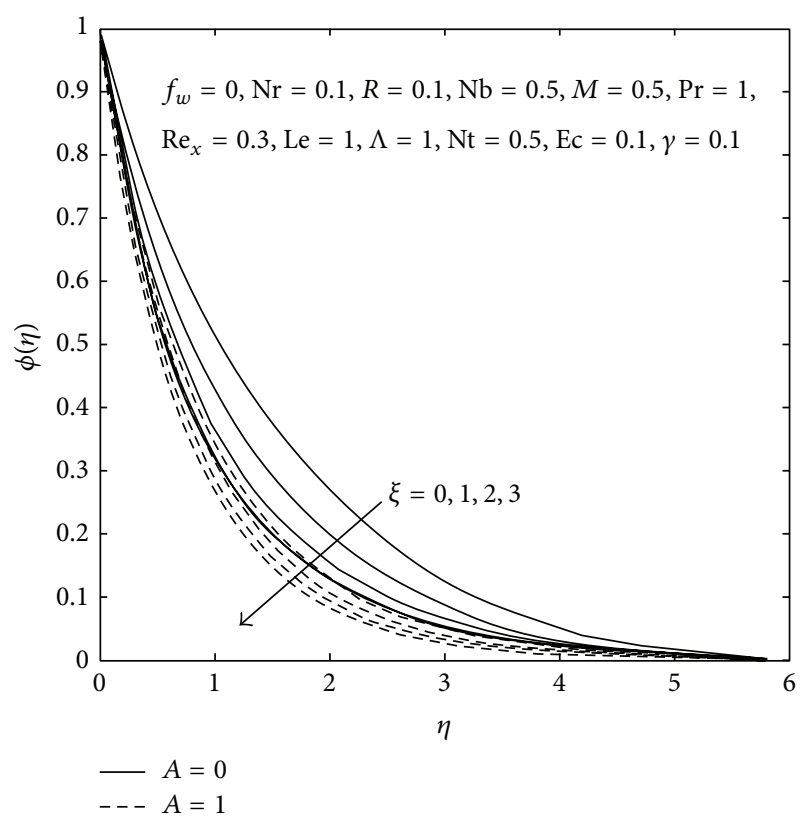

Figure 26: Effects of $\xi$ and $A$ on concentration profile.

profile. In the steady state flow, both parameters moderately enhance the nanoparticles volume fraction. On the other hand, in the case of unsteady flow, the effect of magnetic field on the concentration profile is minimal whereas the effect of buoyancy parameter is almost nil.

Figure 30 shows the effect of radiation parameter on concentration profile. In the case of both steady and unsteady state situations, increment of the radiation parameter minimizes the nanoparticles volume fraction and soon after a certain distance from the boundary the concentration

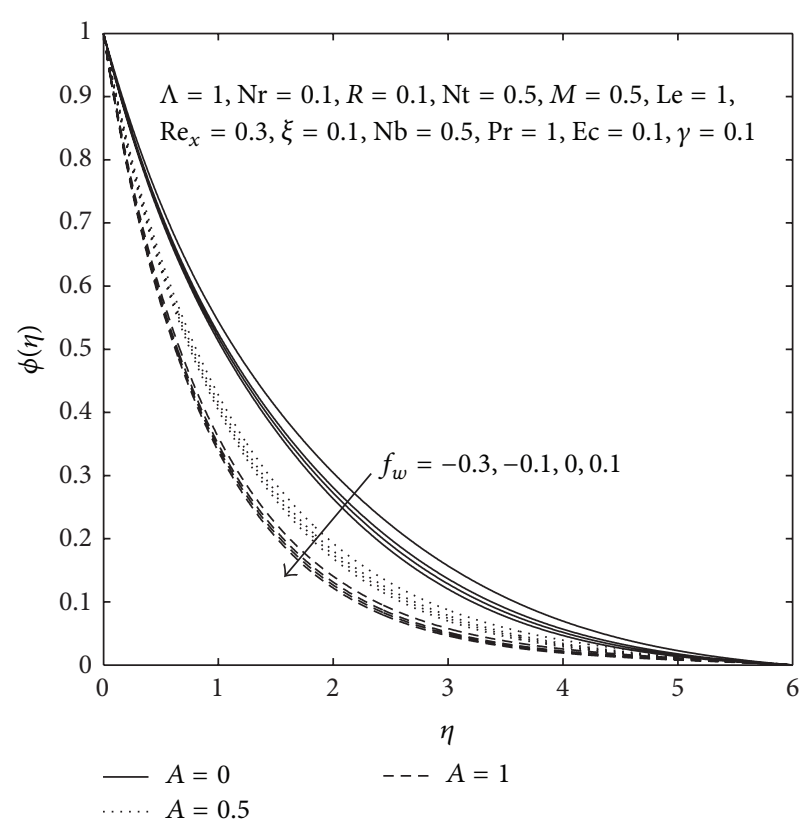

FIgURE 27: Effects of $f_{w}$ and $A$ on concentration profile.

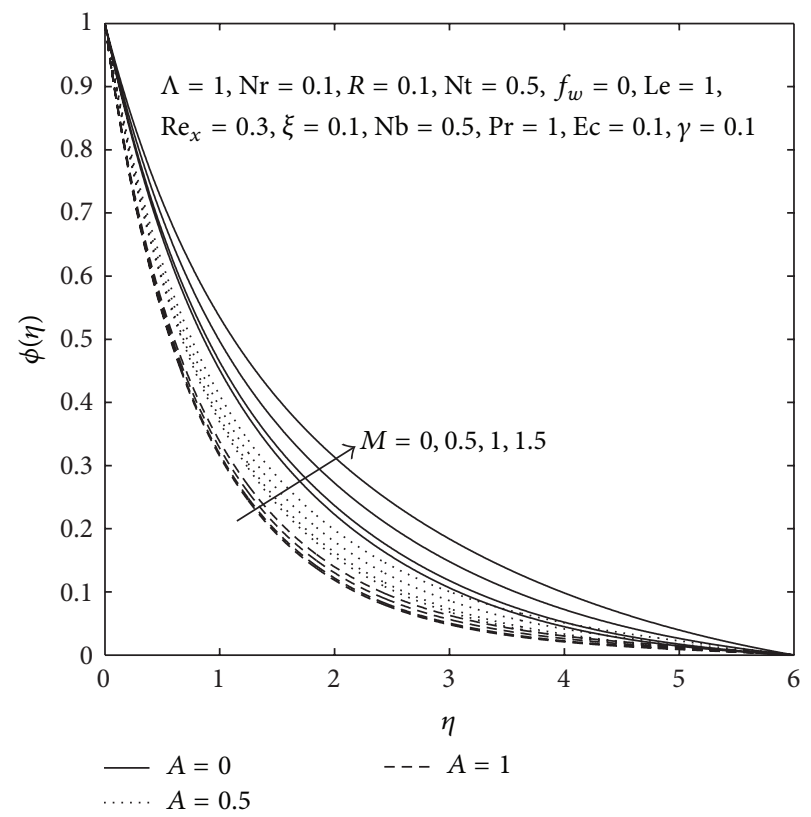

Figure 28: Effects of $M$ and $A$ on concentration profile.

profile remains constant. Moreover, in the case of steady state situation, the heat source parameter significantly decreases the concentration profile near the boundary layer while the effect of this parameter far from the boundary in both steady and unsteady cases has negligible effect on concentration (see Figure 31).

Table 2 shows the effect of unsteady, free convection, buoyancy ratio, and magnetic parameters on skin friction coefficient $-f^{\prime \prime}(0)$, wall heat transfer rate $-g^{\prime}(0)$, and wall mass transfer rate- $h^{\prime}(0)$. Increasing the value of unsteady 


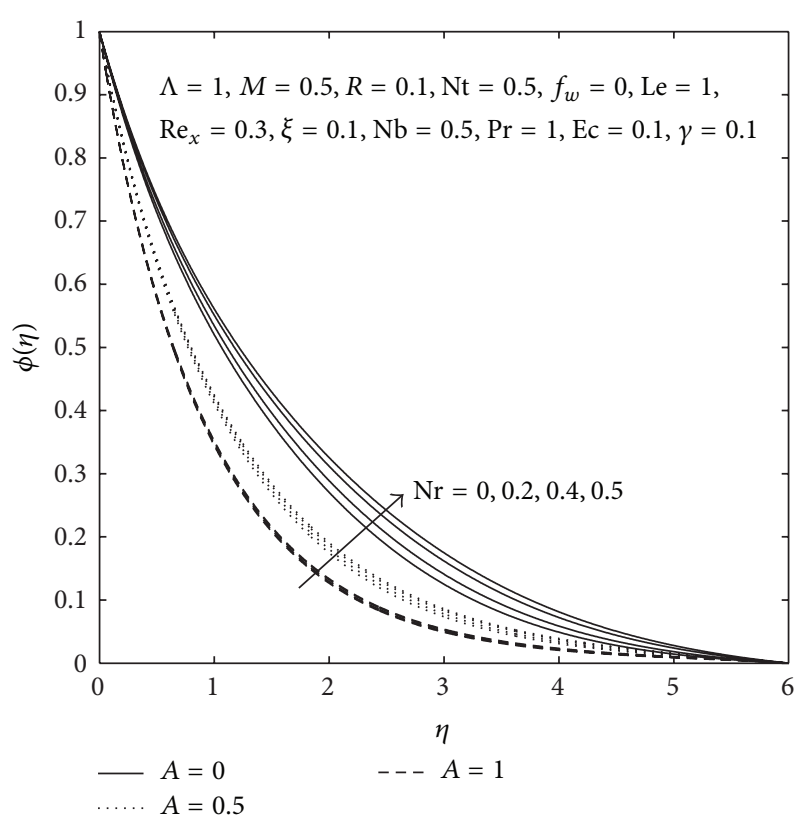

Figure 29: Effects of $\mathrm{Nr}$ and $A$ on concentration profile.

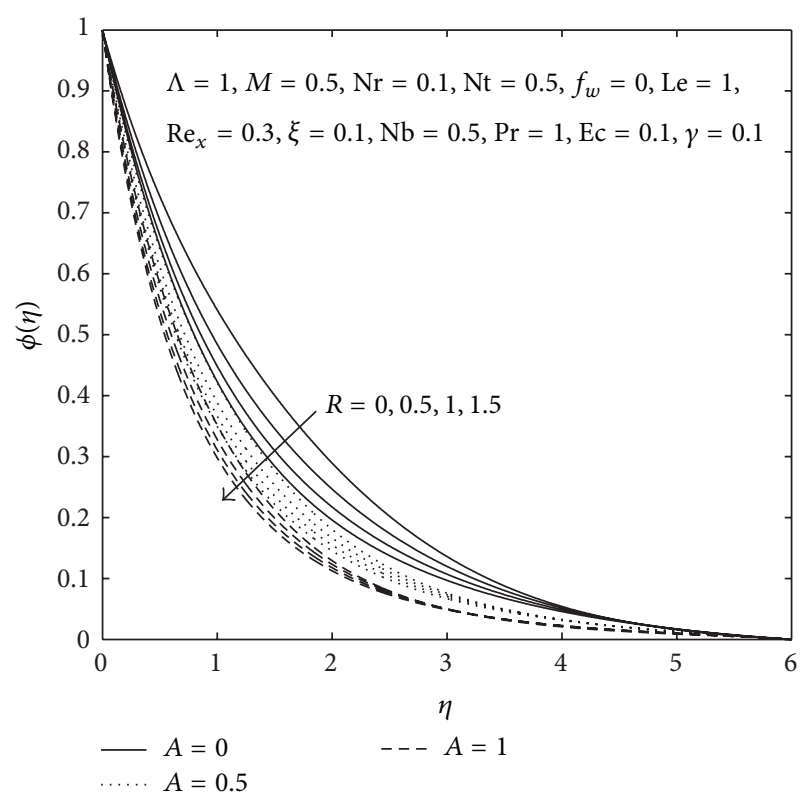

Figure 30: Effects of $R$ and $A$ on concentration profile.

parameter increases all the skin friction coefficient, wall heat transfer rate, and wall mass transfer rate. On the other hand, free convection parameter enhances both wall mass and wall heat transfer rates while it reduces the skin friction coefficient. Both buoyancy ratio and magnetic parameters retard heat transfer and mass transfer rates whereas these parameters enhance the skin friction coefficient.

Effects of suction, thermal radiation, and Brownian motion parameters on skin friction, heat transfer, and mass transfer rates are shown in Table 3. It is observed that the enhanced values of suction parameter lead to increase skin

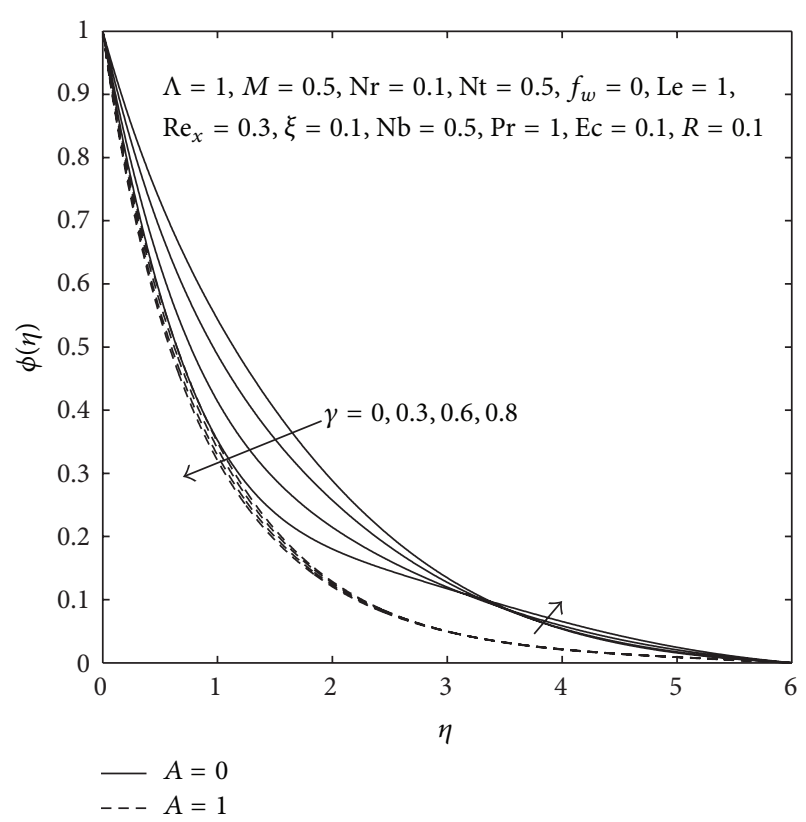

FIGURE 31: Effects of $\gamma$ and $A$ on concentration profile.

friction, heat transfer, and mass transfer rates. On the other hand, both radiation, and Brownian motion parameters enhance mass transfer rate while these parameters reduce both skin friction and heat transfer rate.

Table 4 shows influences of thermophoresis parameter, Prandtl number, and heat source parameters on skin friction, Nusselt number, and Sherwood number. Thermophoresis parameter reduces skin friction, heat transfer, and mass transfer rates. However, increasing the values of Prandtl number leads to increase both skin friction coefficient and heat transfer rate while it decreases mass transfer rate. This is due to the fact that the higher the Prandtl number the thinner the thermal boundary layer and the thicker the nanoparticle volume fraction boundary layer (refer to Figures 20 and 24). As a result of this, the rate of heat diffusion increases while the rate of mass diffusion decreases with increasing values of Pr. On the other hand, increasing the values of heat source parameter results in reducing both the skin friction coefficient and heat transfer rate whereas it makes mass transfer rate increase more.

Table 5 shows the effect of Lewis number, viscous dissipation parameter, and chemical reaction parameter on skin friction, wall heat, and wall mass transfer rates. As it is clearly shown, Eckert number and Lewis number reduce both skin friction and heat transfer rate while both parameters increases mass transfer rate. However, chemical reaction parameter retards both the skin friction coefficient and the heat transfer rate whereas it enhances mass transfer rate.

\section{Conclusion}

The problem of heat and mass transfer in the boundary layer of unsteady viscous nanofluid along a vertical stretching sheet has been studied. The nonlinear governing equations 
associated to the boundary conditions were transformed into coupled ODEs with the help of similarity transformation equations. The solutions of these problems are solved numerically with the help of the Keller box method. The following are some of important results among many:

(i) The velocity, temperature, and concentration profiles of the unsteady flow are less than the corresponding parts of the steady state flow scenario.

(ii) The velocity profile decreases with an increase in the buoyancy ratio number, magnetic parameter, suction parameter, and Prandtl number whereas it increases with the increment of free convection, radiation, Brownian motion, thermophoresis, and heat source parameters.

(iii) Viscous dissipation, thermal radiation, Brownian motion, buoyancy ratio, heat source, and magnetic and thermophoresis parameters enhance the temperature profile whereas Prandtl number, suction parameter, and free convection parameter reduce the temperature profile.

(iv) Nanoparticles volume fraction is enhanced by Prandtl number, thermophoresis parameter, magnetic parameter, and buoyancy ratio number whereas Lewis number, heat source parameter, Brownian motion parameter, radiation parameter, chemical reaction parameter, suction parameter, and free convection parameter reduce the concentration profile.

(v) The skin friction coefficient, heat transfer, and mass transfer rates of unsteady flow are greater than the corresponding parts of the quantities in the steady state situations.

(vi) Increasing the values of the buoyancy ratio number, magnetic parameter, suction parameter, and Prandtl number enhances the skin friction coefficient whereas it decreases with increasing values of free convection, Brownian motion, radiation, thermophoresis, chemical reaction, heat source, viscous dissipation parameters, and Lewis number.

(vii) The presence of Lewis number, thermal radiation, Brownian motion, heat source, magnetic, buoyancy ratio, chemical reaction, thermophoresis, and viscous dissipation parameters in the flow field is to reduce the rate of thermal boundary layer thickness whereas Prandtl number, suction parameter, and free convection parameter maximize the thermal boundary layer thickness.

(viii) The wall mass transfer rate is an increasing function of Lewis number, free convection, chemical reaction, viscous dissipation, suction, Brownian motion, heat source, and radiation parameters while Prandtl number, thermophoresis, and magnetic and buoyancy ratio parameters reduce the mass transfer rate at the plate surface.

\section{Nomenclature}

A: Unsteady parameter

$B$ : Magnetic field strength

C: Nanoparticle concentration

$C_{f}$ : Skin-friction coefficient

$C_{w}$ : Nanoparticles concentration at the stretching surface

$C_{\infty}$ : Nanoparticle concentration far from the sheet

$C_{p}:$ Specific heat capacity at constant pressure

$D_{B}$ : Brownian diffusion coefficient

$D_{T}$ : Thermophoresis diffusion coefficient

Q: Dimensional heat generation coefficient

Ec: Eckert number

$f$ : Dimensionless stream function

$g^{*}$ : Acceleration due to gravity

g: Dimensionless temperature

$h$ : Dimensionless concentration

$k^{*}$ : Rosseland mean absorption coefficient

$k_{f}$ : Thermal conductivity of the base fluid

$k_{p}$ : Thermal conductivity of the nanoparticles

$K_{r}$ : Chemical reaction parameter

Le: Lewis number

M: Magnetic parameter

$\mathrm{Nb}$ : Brownian motion parameter

Nr: Buoyancy ratio number

Nt: Thermophoresis parameter

$\mathrm{Nu}_{x}$ : Nusselt number

Pr: Prandtl number

$R: \quad$ Radiation parameter

$\mathrm{Re}_{x}$ : Local Reynolds number

$\mathrm{Sh}_{x}$ : Sherwood number

$T$ : $\quad$ Fluid temperature

$T_{w}$ : Temperature at the surface

$T_{\infty}$ : Temperature of the fluid far away from the stretching sheet

$u, v$ : Velocity components in the $x$ - and $y$-axes, respectively

$U_{w}$ : Velocity of the wall along the $x$-axis

$x ; y$ : Cartesian coordinates measured along the stretching sheet.

\section{Greek Symbols}

$\alpha$ : Thermal diffusivity of the base fluid

$\beta$ : Thermal expansion coefficient

$\sigma$ : Electrical conductivity

$\sigma^{*}$ : Stefan-Boltzmann constant

$\psi$ : Stream function

$\eta$ : Dimensionless similarity variable

$\Lambda$ : Free convection parameter

$\xi$ : Scaled chemical reaction parameter

$\gamma$ : Heat source parameter

$\mu$ : Dynamic viscosity of the base fluid

$v$ : Kinetic viscosity of the base fluid

$\rho_{f}$ : Density of the base fluid

$\rho_{p}$ : Density of the nanoparticle 
$\tau$ : $\quad$ The ratio of the nanoparticle heat capacity and the base fluid heat capacity

$\left(\rho C_{p}\right)_{f}$ : Heat capacitance of the base fluid $\left(\rho C_{p}\right)_{p}$ : Heat capacitance of the nanoparticle.

\section{Conflict of Interests}

The authors declare that there is no conflict of interests regarding the publication of this paper.

\section{Acknowledgments}

The authors highly appreciate the reviewers of this paper for their critical comments and suggestions given to the authors sparing much of their precious time.

\section{References}

[1] H. S. Takhar, A. J. Chamkha, and G. Nath, "Unsteady threedimensional MHD-boundary-layer flow due to the impulsive motion of a stretching surface," Acta Mechanica, vol. 146, no. 12, pp. 59-71, 2001.

[2] N. Bachok, A. Ishak, and I. Pop, "Boundary-layer flow of nanofluids over a moving surface in a flowing fluid," International Journal of Thermal Sciences, vol. 49, no. 9, pp. 1663-1668, 2010.

[3] O. D. Makinde and A. Aziz, "Boundary layer flow of a nanofluid past a stretching sheet with a convective boundary condition," International Journal of Thermal Sciences, vol. 50, no. 7, pp. 13261332, 2011.

[4] P. O. Olanrewaju, M. A. Olanrewaju, and A. O. Adesanya, "Boundary layer flow of nanofluids over a moving surface in a flowing fluid in the presence of radiation," International Journal of Applied Science and Technology, vol. 2, no. 1, pp. 274-282, 2012.

[5] C.-H. Chen, "Laminar mixed convection adjacent to vertical, continuously stretching sheets," Heat and Mass Transfer, vol. 33, no. 5-6, pp. 471-476, 1998.

[6] K. Vajravelu, K. V. Prasad, and C.-O. Ng, "Unsteady convective boundary layer flow of a viscous fluid at a vertical surface with variable fluid properties," Nonlinear Analysis: Real World Applications, vol. 14, no. 1, pp. 455-464, 2013.

[7] T. S. Chen and F. A. Strobel, "Buoyancy effects in boundary layer adjacent to a continuous moving horizontal flat plate," Journal of Heat Transfer, vol. 102, no. 1, pp. 170-172, 1980.

[8] M. V. Karwe and Y. Jaluria, "Numerical simulation of thermal transport associated with a continuously moving flat sheet in materials processing," Journal of Heat Transfer, vol. 113, no. 3, pp. 612-619, 1991.

[9] M. E. Ali, "The buoyancy effects on the boundary layers induced by continuous surfaces stretched with rapidly decreasing velocities," Heat and Mass Transfer, vol. 40, no. 3-4, pp. 285-291, 2004.

[10] P. Dulal and M. Hiranmoy, "Non-Darcian buoyancy driven heat and mass transfer over a stretching sheet in a porous medium with radiation and ohmic heating," International Journal of Nonlinear Science, vol. 14, no. 1, pp. 115-123, 2012.

[11] E. M. Abo-Eldahab and M. A. El Aziz, "Blowing/suction effect on hydromagnetic heat transfer by mixed convection from an inclined continuously stretching surface with internal heat generation/absorption," International Journal of Thermal Sciences, vol. 43, no. 7, pp. 709-719, 2004.

[12] M. Ali and F. Al-Yousef, "Laminar mixed convection from a continuously moving vertical surface with suction or injection," Heat and Mass Transfer, vol. 33, no. 4, pp. 301-306, 1998.

[13] M. Ali and F. Al-Yousef, "Laminar mixed convection boundary layers induced by a linearly stretching permeable surface," International Journal of Heat and Mass Transfer, vol. 45, no. 21, pp. 4241-4250, 2002.

[14] M. S. Khan, I. Karim, L. E. Ali, and A. Islam, "Unsteady MHD free convection boundary-layer flow of a nanofluid along a stretching sheet with thermal radiation and viscous dissipation effects," International Nano Letters, vol. 2, article 24, 2012.

[15] T. Poornima and N. Bhaskar Reddy, "Radiation effects on MHD free convective boundary layer flow of nanofluids over a nonlinear stretching sheet," Advances in Applied Science Research, vol. 4, no. 2, pp. 190-202, 2013.

[16] M. A. Hamad, I. Pop, and A. I. Ismail, "Magnetic field effects on free convection flow of a nanofluid past a vertical semi-infinite flat plate," Nonlinear Analysis. Real World Applications, vol. 12, no. 3, pp. 1338-1346, 2011.

[17] V. Rajesh, "Radiation effects on MHD free convective flow near a vertical plate with ramped wall temperature," International Journal of Applied Mathematics and Mechanics, vol. 6, no. 21, pp. 60-77, 2010.

[18] A. Raptis, "Free convective oscillatory flow and mass transfer past a porous plate in the presence of radiation for an optically thin fluid," Thermal Science, vol. 15, no. 3, pp. 849-857, 2011.

[19] K. Jafar, R. Nazar, A. Ishak, and I. Pop, "MHD flow and heat transfer over stretching/shrinking sheets with external magnetic field, viscous dissipation and Joule effects," The Canadian Journal of Chemical Engineering, vol. 90, no. 5, pp. 1336-1346, 2012.

[20] M. Gnaneswara Reddy, "Influence of thermal radiation, viscous dissipation and hall current on MHD convection flow over a stretched vertical flat plate," Ain Shams Engineering Journal, vol. 5, no. 1, pp. 169-175, 2014.

[21] K. Vajravelu and A. Hadjinicolaou, "Heat transfer in a viscous fluid over a stretching sheet with viscous dissipation and internal heat generation," International Communications in Heat and Mass Transfer, vol. 20, no. 3, pp. 417-430, 1993.

[22] F. M. Hady, F. S. Ibrahim, S. M. Abdel-Gaied, and M. R. Eid, "Radiation effect on viscous flow of a nanofluid and heat transfer over a nonlinearly stretching sheet," Nanoscale Research Letters, vol. 7, article 229, 2012.

[23] A. Postelnicu, "Influence of chemical reaction on heat and mass transfer by natural convection from vertical surfaces in porous media considering Soret and Dufour effects," Heat and Mass Transfer, vol. 43, no. 6, pp. 595-602, 2007.

[24] M. Shakhaoath Khan, I. Karim, and M. Sirajul Islam, "Possessions of chemical reaction on MHD heat and mass transfer nanofluid flow on a continuously moving surface," The American Chemical Science Journal, vol. 4, no. 3, pp. 401-415, 2014.

[25] R. Kandasamy and P. G. Palanimani, "Effects of chemical reactions, heat, and mass transfer on nonlinear magnetohydrodynamic boundary layer flow over a wedge with a porous medium in the presence of ohmic heating and viscous dissipation," Journal of Porous Media, vol. 10, no. 5, pp. 489-501, 2007.

[26] L. J. Grubka and K. M. Bobba, "Heat transfer characteristics of a continuous, stretching surface with variable temperature," Journal of Heat Transfer, vol. 107, no. 1, pp. 248-250, 1985. 
[27] A. Ishak, R. Nazar, and I. Pop, "Boundary layer flow and heat transfer over an unsteady stretching vertical surface," Meccanica, vol. 44, no. 4, pp. 369-375, 2009.

[28] K. Vendabai and G. Sarojamma, "Unsteady convective boundary layer flow of a nanofluid over a stretching surface in the presence of a magnetic field and heat generation," International Journal of Emerging Trends in Engineering and Development, vol. 3, no. 4, pp. 214-230, 2014.

[29] J. Buongiorno, "Convective transport in nanofluids," Journal of Heat Transfer, vol. 128, no. 3, pp. 240-250, 2006.

[30] F. M. Hady, R. E. Mohamed, and M. A. Ahmed, "A nanofluid flow in a non-linear stretching surface saturated in a porous medium with yield stress effect," Applied Mathematics \& Information Sciences Letters, vol. 2, no. 2, pp. 43-51, 2014.

[31] T. Cebeci and P. Bradshaw, Physical and Computational Aspects of Convective Heat Transfer, Springer, New York, NY, USA, 1988.

[32] M. Goyal and R. Bhargava, "Numerical solution of MHD viscoelastic nanofluid flow over a stretching sheet with partial slip and heat source/sink," ISRN Nanotechnology, vol. 2013, Article ID 931021, 11 pages, 2013. 

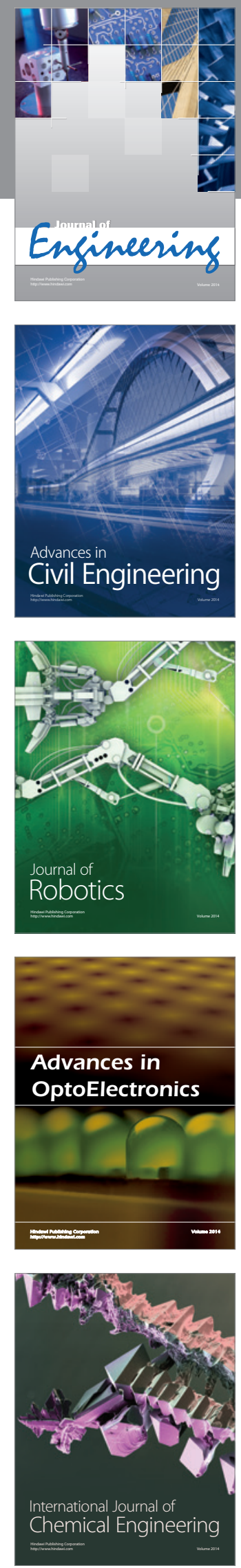

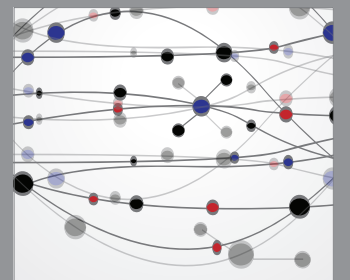

The Scientific World Journal
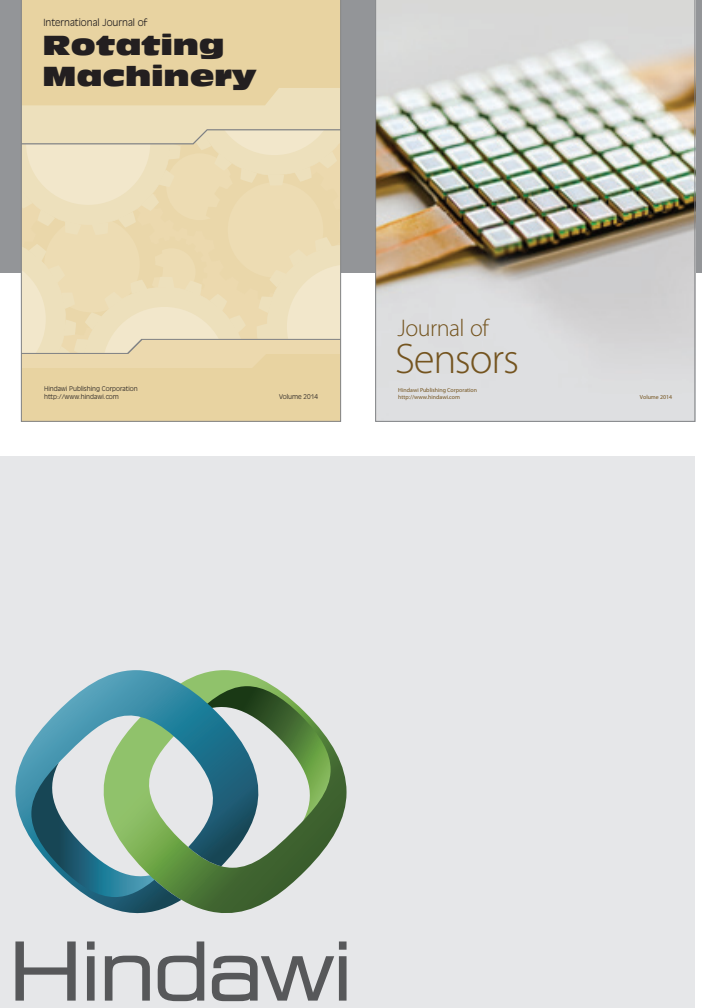

Submit your manuscripts at http://www.hindawi.com
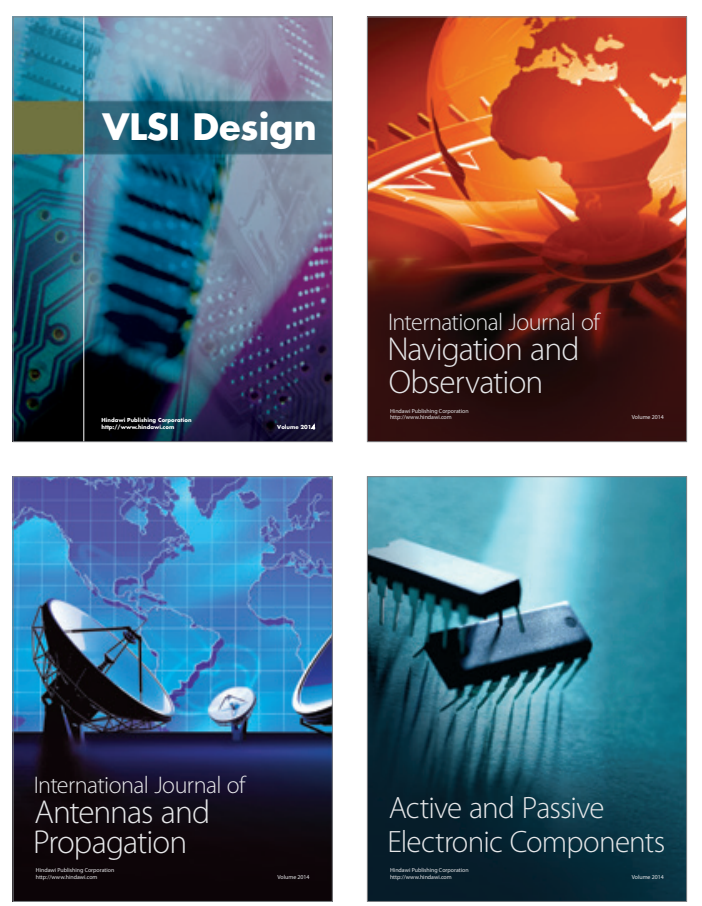
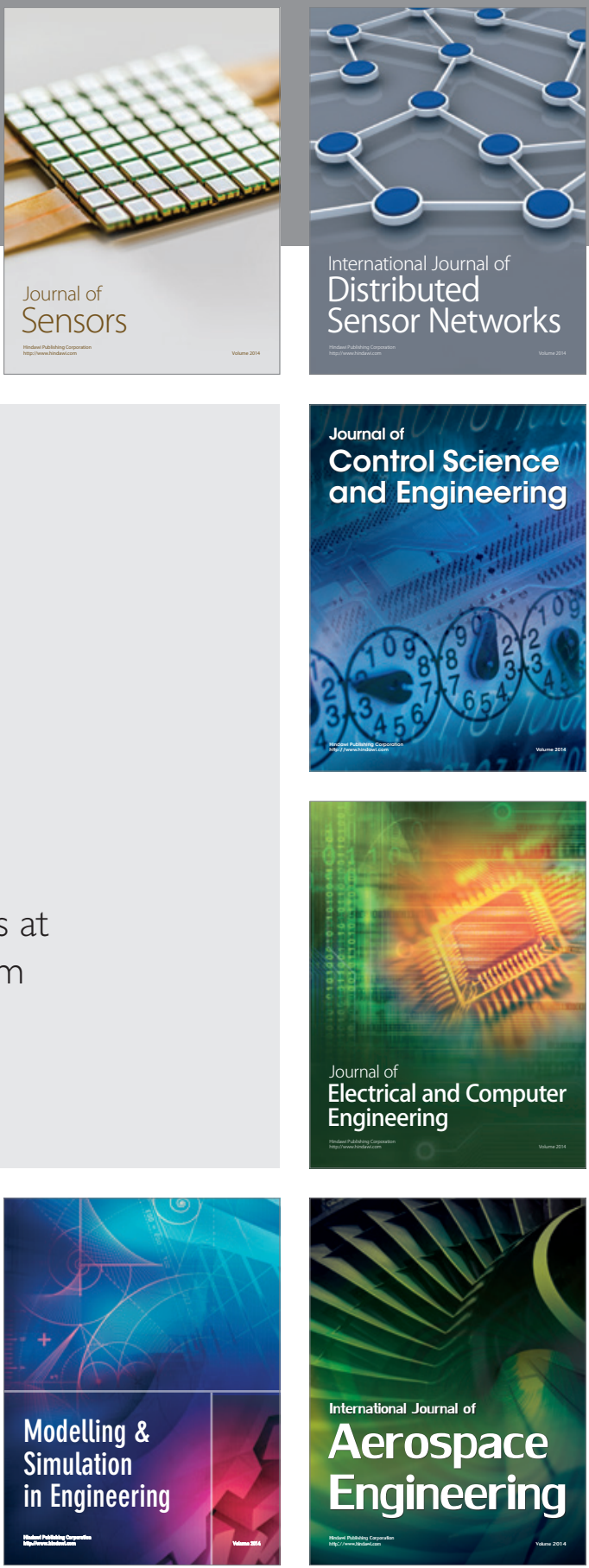

Journal of

Control Science

and Engineering
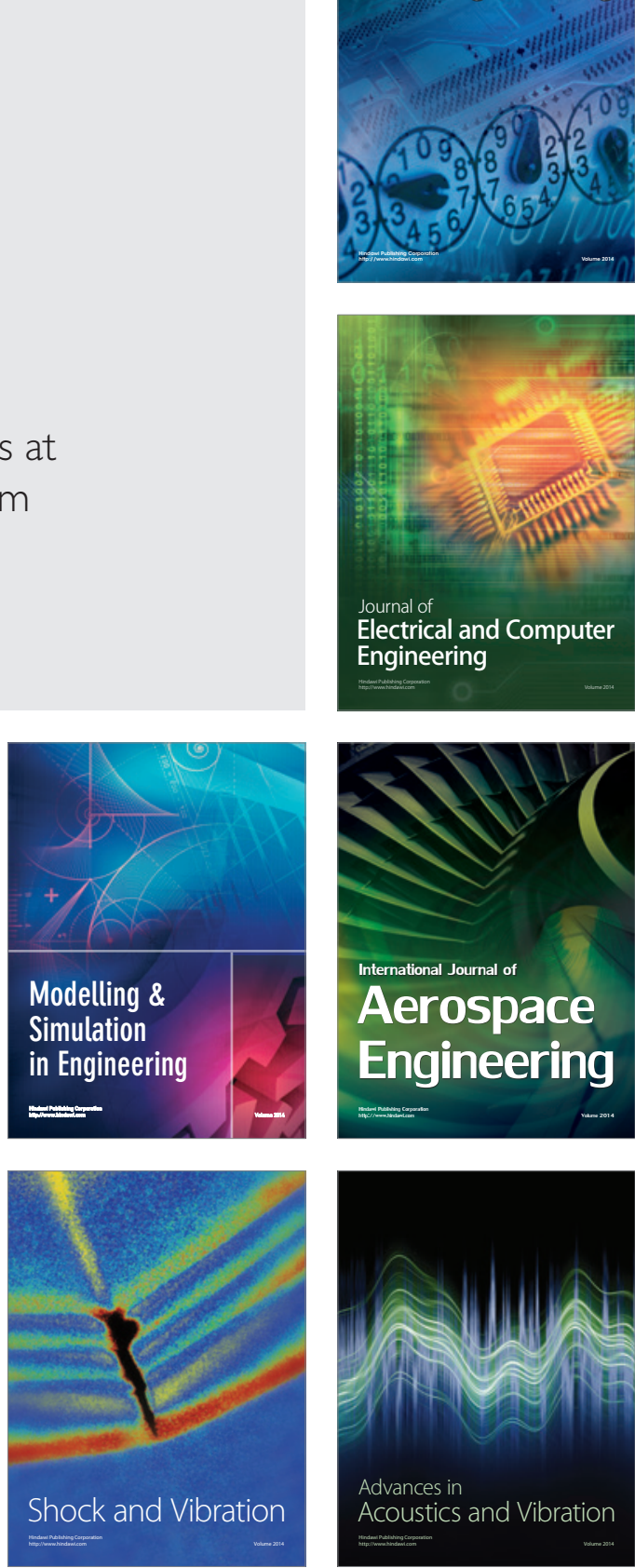